\title{
Alpha-Synuclein is a Target of Fic-mediated Adenylylation/AMPylation: Implications for Parkinson's Disease
}

\author{
Anwesha Sanyal ${ }^{\mathrm{a} \mathrm{g}^{*}}$, Sayan Dutta ${ }^{\mathrm{b}^{*}}$, Aswathy Chandran ${ }^{\mathrm{b}}$, Antonius Koller ${ }^{\mathrm{c}, \mathrm{h}}$, Ali Camara ${ }^{\mathrm{a}}$, Ben G. \\ Watson $^{a}$, Ranjan Sengupta ${ }^{a}$, Daniel Ysselstein ${ }^{b, c}$, Paola Montenegro ${ }^{b, c}$, Jason Cannon ${ }^{d}$, Jean- \\ Christophe Rochet ${ }^{\mathrm{b}, \mathrm{e}}$, and Seema Mattoo ${ }^{\mathrm{a}, \mathrm{f}, 1}$ \\ ${ }^{a}$ Department of Biological Sciences, ${ }^{b}$ Department of Medicinal Chemistry and Molecular \\ Pharmacology, Purdue University, 'Herbert Irving Comprehensive Cancer Center, Columbia \\ University Medical Center, New York, NY, 'School of Health Sciences, Purdue University,

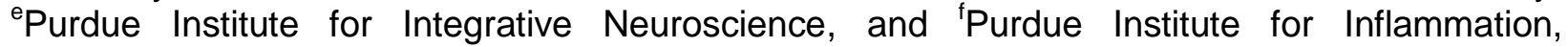 \\ Immunology and Infectious Disease, Purdue University, 915 W State St., LILY G-227, West \\ Lafayette, IN 47907 \\ *equal contribution \\ ${ }^{1}$ To whom correspondence should be addressed. E-mail: smattoo@purdue.edu \\ 'Present address: Ann Romney Center for Neurologic Diseases, Brigham and Women's \\ Hospital, Harvard Medical School, Boston, MA \\ hPresent address: Northeastern University, Barnette Institute, Boston, MA \\ The authors declare no conflict of interest.
}




\section{HIGHLIGHTS:}

- Aggregated forms of the presynaptic protein aSyn cause neurotoxicity and induce ER stress in cellular and animal models of Parkinson's disease.

- We have identified aSyn as a novel target for the human Fic protein, HYPE, a key regulator of ER homeostasis.

- HYPE adenylylates aSyn and reduces the aggregation of recombinant aSyn

- Fic-mediated adenylylation/AMPylation is a possible mechanism by which cells cope with aSyn toxicity. 
Sanyal \& Dutta et al., 2019 - Graphic Abstract

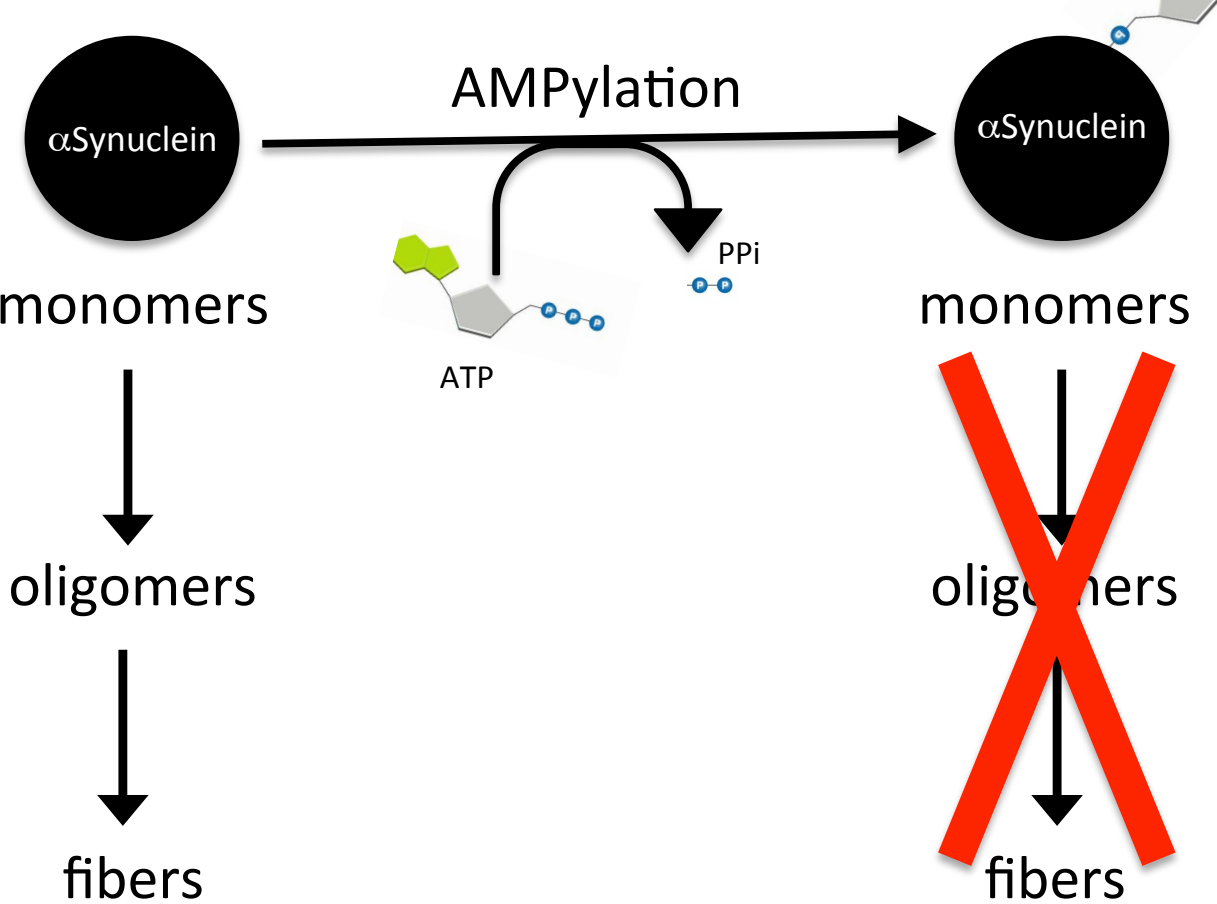




\begin{abstract}
:
During disease, cells experience various stresses that manifest as an accumulation of misfolded proteins and eventually lead to cell death. To combat this stress, cells activate a pathway called UPR (Unfolded Protein Response) that functions to maintain ER (endoplasmic reticulum) homeostasis and determines cell fate. We recently reported a hitherto unknown mechanism of regulating ER stress via a novel post-translational modification (PTM) called Ficmediated Adenylylation/AMPylation. Specifically, we showed that the human Fic (filamentation induced by cAMP) protein, HYPE/FicD, catalyzes the addition of an AMP (adenosine monophosphate) to the ER chaperone, BiP, to alter the cell's UPR-mediated response to misfolded proteins. Here, we report that we have now identified a second target for HYPE alpha-Synuclein (aSyn), a presynaptic protein involved in Parkinson's disease (PD). Aggregated aSyn has been shown to induce ER stress and elicit neurotoxicity in PD models. We show that HYPE adenylylates aSyn and reduces phenotypes associated with aSyn aggregation in vitro, suggesting a possible mechanism by which cells cope with aSyn toxicity.
\end{abstract}




\section{INTRODUCTION:}

Post-translational modifications (PTMs) catalyzed by the Fic (filamentation induced by cAMP) family of enzymes are fast being recognized as a central regulatory paradigm governing diverse signal transduction pathways in both prokaryotes and eukaryotes [1]. Fic proteins consist of a core $\mathrm{HxFx}(\mathrm{D} / \mathrm{E})(\mathrm{G} / \mathrm{A}) \mathrm{N}(\mathrm{G} / \mathrm{K}) \mathrm{R} x \mathrm{xR}$ motif, with the Histidine being essential for catalytic activity [2]. Additionally, the activity of most Fic proteins is inhibited by inter- and intramolecular interactions with an inhibitory helix ( $\alpha$-inh), characterized by an $(\mathrm{S} / \mathrm{T}) \mathrm{xxxE}(\mathrm{G} / \mathrm{N})$ motif [3]. We previously discovered that some bacterially secreted Fic proteins could induce host cell toxicity by covalently modifying mammalian Rho GTPases RhoA, Rac1, and Cdc42 with AMP (adenosine monophosphate) [2], [4]. This adenylylation or "AMPylation" event renders the Rho GTPases inactive, thereby inducing cytoskeletal collapse and allowing the bacteria to evade phagocytosis [5], [6]. We further showed that the activity of Fic proteins was conserved in eukaryotes. Specifically, we demonstrated that the sole Fic protein in humans, HYPE (Huntingtin yeast interacting protein E) or FicD, functions as an adenylyltransferase [2, 4, 7]. HYPE is a $52 \mathrm{kDa}$ protein and is classified as a Class II Fic protein, consisting of a canonical Fic domain and an $\alpha$-inh sequence that lies $\mathrm{N}$-terminal to its Fic motif (Figure 1A). Accordingly, a mutation of HYPE's His363 to Ala renders it enzymatically inactive, while a mutation of its Glu234 to Gly renders it constitutively active [3], [7]. We showed that HYPE localizes to the lumen of the ER (endoplasmic reticulum) and adenylylates the Hsp70 chaperone, BiP (Binding immunoglobulin protein) [7]. BiP monitors protein folding in cells and serves as a sentinel for the activation of an ER stress response pathway called the UPR (unfolded protein response)[8].

During disease, cells experience various stresses that manifest as an accumulation of misfolded proteins and eventually lead to cell death [9]. To combat this stress, cells activate the UPR pathway, which initially functions to restore normalcy to the cell. However, upon sustained stress, the UPR triggers apoptosis. Thus, maintenance of ER homeostasis is a critical aspect of determining cell fate and requires a properly functioning UPR. It was recently reported that while E234G-HYPE adenylylates BiP, WT-HYPE serves to reverse HYPE-mediated adenylylation of BiP [10]. Thus, WT-HYPE is classified as a de-AMPylase and a H363A mutation in the wild type background renders an inactive de-AMPylase. Together, the adenylyltransferase and deAMPylase activity of HYPE on BiP highlights the important role HYPE plays in tightly regulating UPR and ER homeostasis in response to protein misfolding.

Proper protein folding entails a process where the nascent polypeptides are folded into a three-dimensional functional identity. The organized native structure of the protein is at its energy minimum state while the unfolded polypeptide has higher free energy [11]. Molecular chaperones in the cytosol and $\mathrm{ER}$, such as $\mathrm{BiP}$, bind misfolded proteins with varying specificity and mediate their folding [12]. Any changes in the system that destabilize the folding process lead to the formation of misfolded species that lack a thermodynamically stable structure and hence have a propensity to aggregate [13]. These aggregates are prone to further aggregation by a process called nucleation, thus increasing in size at an accelerated rate [13]. Such aggregates are a hallmark of various degenerative diseases like Parkinson's disease (PD), Alzheimer's disease, Huntington's disease, type II diabetes, cystic fibrosis and others [13]. Several lines of evidence suggest that accumulation of specific protein aggregates is toxic for cells [14].

PD is a progressive neurodegenerative disorder characterized by tremors, bradykinesia, rigidity and instability of posture [15]. These motor symptoms are thought to arise due to the toxic accumulation of aggregates of a presynaptic protein - alpha-Synuclein ( $\alpha$ Syn) - in dopaminergic neurons of the substantia nigra in the midbrain [16]. aSyn is encoded by the SNCA gene and consists of a 140 amino acids (Figure 1B). It is an intrinsically disordered protein that consists of an amphipathic $\mathrm{N}$-terminal region and an acidic $\mathrm{C}$-terminal region flanking a highly hydrophobic central domain, also referred to as the NAC (Non-Amyloid-beta Component of Alzheimer's disease) domain. Because of its natively unfolded structure, aSyn 
has a high propensity to aggregate[13]. Further, point mutations in the SNCA gene that target Ala53 in particular and those that result in Ala53Thr, Ala53Glu, Ala30Pro, Glu46Lys, His50GIn, and Gly51Asp substitutions, as well as duplication or triplication of the gene, lead to familial forms of PD due to enhanced aggregation of the protein [17]. Acetylation, methylation, $\mathrm{O}$ GlcNAcylation, and phosphorylation of aSyn are also reported, suggesting possible roles of PTMs in PD [18] [53]. aSyn fibrils accumulate in structures called Lewy bodies in surviving neurons [19]. Although Lewy bodies are a hallmark neuropathological feature of PD, evidence suggests that the intermediate oligomeric species capable of forming fibrils may be more toxic than Lewy bodies, potentially because of their ability to permeabilize lipid membranes [20-23]. Such aSyn oligomers can also diffuse into the ER lumen and interact directly with BiP. Accumulation of aSyn oligomers results in ER stress and activation of UPR in affected neurons $[24,25]$.

Our discovery that HYPE adenylylates BiP established HYPE as a new player in UPR regulation in response to $E R$ stress. We and others have now demonstrated that adenylylation alters BiP's ATPase activity and activation of the downstream UPR cascades to reinstate ER homeostasis [7], [26-28]. Given this critical role for HYPE in how cells cope with stress from misfolded proteins and the direct correlation between aSyn accumulation, ER stress and PD progression, we reasoned that HYPE may play a role in PD, possibly via UPR regulation or direct interaction with aSyn. Indeed, a role for HYPE in neurodegeneration was recently reported in a $C$. elegans model, where activation of HYPE's adenylyltransferase activity was shown to induce neuroprotective aggregative effects of proteins like amyloid beta $(A \beta)$, mutant Huntingtin (m-Htt), and aSyn through its manipulation of cytosolic Hsp70 chaperones [29]. We, too, have shown previously that HYPE can directly bind to misfolded proteins [7], and global proteomic analyses of adenylylated peptides by mass spectrometry indicate targets for HYPE other than $\mathrm{BiP}$ and related heat shock proteins [30, 31]. However, aSyn has never been identified as a target for HYPE or known to be adenylylated.

Here, we report aSyn as a bona fide novel target for HYPE. We show that HYPE directly binds aSyn in vitro and adenylylates it at threonine residues predominantly in its $\mathrm{N}$-terminus. Accordingly, analysis of rat midbrain sections and primary cultures reveals that HYPE is enriched in neurons of the substantia nigra and colocalizes with markers for dopaminergic neurons, sites where aSyn aggregates during PD. Importantly, adenylylation alters the structure of aSyn fibrils and leads to inhibitory effects on aSyn fibrillation and aSyn-mediated membrane permeabilization. Collectively, these results suggest that HYPE-mediated aSyn adenylylation may be a mechanism by which affected cells cope with aSyn toxicity. This is the first report identifying aSyn as a target for HYPE, and reinforces a role for Fic-mediated adenylylation/AMPylation in neurodegeneration. Importantly, our data shed light on a possible direct means of modifying and reducing toxicity from misfolded aggregates, which may function in parallel with HYPE's effect on chaperones.

\section{RESULTS}

\section{HYPE is enriched in the substantia nigra in rat midbrain sections}

Global transcriptomic and proteomic studies indicate that HYPE is expressed at extremely low levels in all tissues, including neurons [2]. Indeed, endogenous HYPE is rarely detected in most epithelial cells like HEK293 or HeLa by standard Western blotting or immunofluorescence techniques [7]. To assess HYPE expression in neurons, we performed immunohistochemistry on sections of rat midbrain using antibody generated against bacterially purified full length human HYPE, where human and rat HYPE (FicD) share $90 \%$ amino acid sequence identity. We observed strong staining for HYPE in sections corresponding to the substantia nigra, a region where neuronal toxicity occurs due to aSyn aggregation during PD 
(Figure 2A). HYPE's staining pattern within the substantia nigra appeared neuronal under higher magnification analysis (Figure 2A, red and blue boxes, and supplemental Figure 1). We also saw evidence of HYPE staining in the pyramidal neurons of the hippocampus (as indicated in Figure 2A).

\section{HYPE is expressed in nigral dopaminergic neurons and localizes to the cell body}

Toxicity induced by aSyn aggregation predominantly affects nigral dopaminergic neurons [15]. We, therefore, assessed the localization of HYPE in primary dissociated neurons derived from embryonic rat midbrain. Immunofluorescence analyses of HYPE revealed that it colocalizes with the neuronal marker MAP2 (microtubule-associated protein 2) as detected by fluorescence microscopy, confirming HYPE expression in neurons (Figure 2B), whereas HYPE expression in surrounding non-neuronal cells (consisting primarily of astrocytes) was much weaker. Interestingly, compared to MAP2, which is expressed across both the cell body and in neurites, HYPE is detected predominantly in the neuronal cell body, but not to a large extent in the dendrites (Figure 2B, upper panels). In contrast to HYPE, the chaperone protein BiP (also an ER lumenal protein) displays ER-specific expression in all parts of the neuron and is present at higher levels in surrounding astrocytes (Figure 2B, lower panels and Supplemental Figure 2). Finally, we observed HYPE expression in dopaminergic neurons that stained positive for tyrosine hydroxylase (TH) (Figure $2 \mathrm{C}$ ), indicating that HYPE is expressed in neurons that are most vulnerable in PD. Together, these data support our hypothesized link between HYPE activity and its relevance to PD.

\section{HYPE interacts with aSyn and adenylylates it in vitro}

aSyn is known to localize to the ER lumen and interact with BiP [24, 32, 33]. We previously reported that HYPE interacts with and adenylylates BiP in the ER lumen, and alters UPR activation presumably by altering BiP's ability to refold misfolded proteins [7]. Additionally, we found that HYPE itself has the ability to bind to misfolded proteins [7]. Thus, aSyn is correctly situated in the ER to allow interactions with HYPE. Given HYPE's role in UPR regulation and its expression in regions of the brain that are associated with aggregation of misfolded aSyn, we asked whether HYPE directly interacts with aSyn. Complementary coimmunoprecipitation experiments using SH-SY5Y neuroblastoma-derived cells with antibody to aSyn or to HYPE revealed a direct interaction between the two proteins (data not shown). Our experience with HYPE informs us that in its enzymatically active state, it interacts transiently with its substrates (like BiP); however, transient interactions can be captured in vitro by assessing interactions using the catalytically dead E234G/H363A-HYPE mutant, which functions as a substrate trap [7]. We, therefore, conducted biolayer interferometry with bacterially expressed and purified His-tagged aSyn and untagged E234G/H363A-HYPE, with aSyn immobilized on the anti-His antibody sensor, to determine HYPE's binding efficiency for aSyn. E234G/H363A-HYPE displayed sustained binding to aSyn with a Kd of $15.3 \pm 0.04 \mu \mathrm{M}$ (Figure 3A).

We next asked if HYPE could adenylylate aSyn. For this, untagged aSyn was purified as oligomeric and as low molecular weight, mostly monomeric species (by passing the aSyn solution prepared by dissolving the lyophilized protein over a $100 \mathrm{kD}$-cut off filter). Monomeric and oligomeric aSyn were incubated with E234G-HYPE or its enzymatically inactive E234G/H363A mutant in an in vitro adenylylation reaction using $\alpha-{ }^{32} \mathrm{P}-\mathrm{ATP}$ as a nucleotide source. Both monomeric and oligomeric forms of aSyn were efficiently adenylylated by enzymatically active E234G-HYPE but not by E234G/H363A HYPE (Figure 3B). Even though equal concentrations of monomeric and oligomeric aSyn were used in this reaction, monomeric aSyn appeared to be more efficiently adenylylated, as determined by stronger intensity of radiolabelled bands corresponding to aSyn on the autoradiogram (Figure 3B). These data are the first indication of aSyn as a novel target for HYPE-mediated adenylylation. 


\section{HYPE adenylylates aSyn at threonine residues predominantly in its $\mathrm{N}$-terminus}

Monomeric aSyn incubated with E234G-HYPE in an in vitro adenylylation reaction as described above was digested with trypsin or chymotrypsin and subjected to LC-MS/MS mass spectrometric analysis (see Methods). Peptides with a mass shift corresponding to the addition of an AMP were found to be modified at amino acids T33, T54, and T75. Representative spectra for peptides with an AMP modification at T33, T54, and T75 showing peaks at $\mathrm{m} / \mathrm{z} 348.08$ representing the protonated form of $\mathrm{AMP}$ and neutral losses from the peptides of adenine, adenosine or AMP are shown in Figures 4A, B, and C. Interestingly, nearly all the aSyn adenylylation sites identified lie in the $\mathrm{N}$-terminus domain, a region critical for interacting with the membrane and needed for stabilizing aSyn [34].

\section{Adenylylation of aSyn shows inhibitory effects on aggregation and fibril formation}

As mentioned earlier, aSyn aggregation is thought to play a key role in neurodegeneration associated with PD. Therefore, to determine the functional consequence of aSyn adenylylation, we asked whether adenylylation affects the kinetics of aSyn fibril formation. To monitor fibrillation kinetics, we conducted an in vitro aSyn fibrillation assay using thioflavin T (ThT), a fluorescent dye that forms a complex with the aggregating aSyn protein. ThT fluorescence intensity directly correlates with the extent of fibrillation. Mouse WT aSyn and a human A53T aSyn clinical mutant, which are known to display an increased rate of fibrillation, were used as positive controls [35]. Data from representative fibrillation assays are presented in Figure 5A and in supplemental Figure 3. aSyn alone or first adenylylated by E234G-HYPE was allowed to incubate with ThT under shaking conditions to induce the formation of fibrils and monitored for 5 days. As expected, untreated WT-aSyn formed aggregates reaching saturation levels, as evidenced by a sigmoidal fluorescence trace with a distinct lag phase and plateau (Figure 5A, blue line). In contrast, data from five of the seven biological replicates showed that fibril formation by aSyn adenylylated by E234G-HYPE was significantly compromised, displaying markedly reduced ThT fluorescence levels (Figure 5A; compare blue line vs green line). aSyn left unmodified by incubating with catalytically inactive E234G/H363A-HYPE did not show impaired fibrillation, indicating that the mere presence of HYPE does not affect the fibrillation process (Figure 5A; purple line). Interestingly, incubation of aSyn with WT-HYPE also displayed a slight reduction in fibrillation levels in some biological replicates (Figure 5A, red line and supplementary Figure 3). Quantification of the end-point fibrillation levels, however, indicated that aSyn fibrillation levels were significantly reduced only upon incubation with the adenylylation-competent E234G-HYPE (Figure 5B).

The fibrillation results were verified by separating and quantifying the insoluble pellet (aggregated aSyn fibrils) and supernatant (soluble, monomeric aSyn) fractions from each of the fibrillation samples. Specifically, equal amounts of end-point fibrillation samples were subjected to ultracentrifugation. The supernatant $(S)$ and pellet $(P)$ fractions were separated by SDSPAGE and analyzed by western blotting with antibody to aSyn, to distinguish the monomeric and oligomeric aSyn proteins (Figure 5C). Quantification of monomeric aSyn in each of these fractions revealed that adenylylation significantly reduced the amount of insoluble aSyn (Figure $5 \mathrm{D})$. Depicted in Fig 5D is the quantification of monomeric aSyn in "Pellet" fractions normalized against the corresponding aSyn only (100\%) sample. The histogram corresponding to adenylylated aSyn shows decreased insolubility of $74 \%$. Interestingly, assessment of the fibrillation data shown in Figure 5A normalized against the maximum fluorescence for each individual sample revealed a similar lag time for all samples incubated with HYPE when compared to aSyn alone (Figure 5E), whereas the lag times observed for the A53T and mouse aSyn positive controls were markedly shorter. 


\section{Adenylylation alters aSyn fibril morphology}

Next, we examined the end-point fibrillation samples shown in Figure 5A by transmission electron microscopy (TEM) to confirm that amyloid-like fibrils were formed by WT and HYPEtreated aSyn, and to assess whether adenylylation had any effect on aSyn fibril structure. All the samples displayed an unbranched fibril morphology (Figure 6). In agreement with the literature [36], WT- $\alpha$ Syn fibrils were most frequently arranged as two fibrils (each with a diameter of $\sim 10$ $15 \mathrm{~nm}$ ) wound around each other via a helical twist characterized by a well-defined pitch length (Figures 6A). Fibrils with a similar twisted morphology were also observed in samples of aSyn incubated with adenylylation-incompetent WT-HYPE or E234G/H363-HYPE (Figures 6B and 6C, red arrows). In contrast, aSyn adenylylated by E234G-HYPE displayed a mixed population of fibrils: whereas some paired fibrils had a twisted morphology (red arrows), others were arranged in a parallel fashion without any twist (yellow arrows) (Figure 6D). These data suggest that aSyn adenylylation can interfere with the protein's ability to form typical twisted fibrils under the conditions used here, possibly due to steric hindrance from the adenylylation PTM.

\section{Adenylylation reduces membrane permeabilization}

The pathological mechanism of action and physiological role of aSyn are poorly understood. Interaction with membrane surfaces has been shown to promote the process of self-aggregation and lead to disruption of bilayer integrity by lipid extraction, phenomena which could play a central role in aSyn neurotoxicity [34, 37]. We have also observed that familial mutants of aSyn that show greater neurotoxicity in primary neuronal cell cultures (e.g. A30P and G51D) also display greater vesicle disruption [34, 37]. Thus, we asked whether adenylylation alters aSyn's ability to permeabilize the membrane. To this end, we compared unmodified WT aSyn to that incubated with WT-, E234G-, or E234G/H363A-HYPE for their ability to disrupt small unilamellar vesicles (SUV) using a calcein dye release assay. SUVs prepared from a 50:50 mixture $(\mathrm{mol} / \mathrm{mol})$ of egg phosphatidylglycerol $(P G)$ and egg phosphatidylcholine (PC) were loaded with calcein at a self-quenching concentration and incubated with the adenylylated or unmodified aSyn variants. Permeabilization was assessed by real time measurement of the increase in fluorescence intensity caused by dequenching of the released dye into the surrounding buffer. Interestingly, aSyn adenylylated by E234G-HYPE showed a significant decrease in vesicle leakage compared to the non-adenylylated samples (Figure 7), suggesting that adenylylation may be a protective mechanism to combat the toxic effects of vesicle disruption associated with membrane-induced aSyn aggregation.

\section{DISCUSSION}

The discovery of Fic proteins as evolutionarily conserved adenylyltransferases has brought about a renewed interest in adenylylation/AMPylation, and underscores the importance of this previously underappreciated post-translational modification (PTM). Documentation of adenylylation in signal transduction is growing. Fic proteins represent one of three known types of adenylyltransferases, the other two being bacterial glutamine synthase adenylyltransferase (GS-AT) and the newly discovered Sel-O pseudokinase [38, 39]. PTMs catalyzed by Fic proteins are now being recognized as a universal signaling paradigm that regulates critical processes like bacterial pathogenesis, cellular trafficking, drug tolerance, and prokaryotic and eukaryotic stress response [1]. Further, our discovery of BiP as the elusive target for the human Fic protein, HYPE, has unveiled Fic-mediated adenylylation as a critical step in UPR regulation and the cell's ability to cope with misfolded proteins [7]. Further, through its ability to manipulate 
chaperone activity, Fic-mediated adenylylation has recently been linked to neurodegeneration [29].

As mentioned earlier, HYPE localizes to the ER lumen. To date, BiP is the only known ER-localized physiological target for HYPE. However, some cellular and proteomic analyses have identified additional targets - such as histones and cytosolic Hsp70 and Hsp40 family chaperones - that localize to cellular compartments other than the ER [30], [31], [40]. How HYPE interacts physiologically with these non-ER localized proteins is unclear [54], though a recent report reveals that a mass exodus of $E R$ resident proteins occurs in response to $E R \mathrm{Ca}^{2+}$ depletion [41]. Additionally in C. elegans, HYPE is reported to be partially present in the cytosol in addition to the ER lumen [29].

Here, we identify aSyn, a presynaptic protein involved in Parkinson's disease pathogenesis, as a novel target for the human Fic protein, HYPE, and propose a direct role for adenylylation in modulating neurotoxicity. During PD, accumulation and aggregation of misfolded aSyn manifests as the formation of Lewy bodies and is thought to lead to the death of dopaminergic neurons in the substantia nigra region of the brain, potentially via a mechanism involving vesicle disruption. Accordingly, we see that HYPE is present in dopaminergic neurons and enriched in the substantia nigra of normal rat brains (Figure 2). While aSyn is predominantly a cytosolic protein, it is known to transgress membranes upon oligomerization [24, 37]. aSyn has been reported to enter the ER lumen, where it interacts with BiP and is suggested to trigger a UPR response [24]. Thus, we predict that HYPE and aSyn likely interact directly in the ER lumen. Alternatively, released cytosolic pools of HYPE may interact with aSyn. Indeed, we confirmed a direct interaction between HYPE and aSyn using immunoprecipitation and biolayer interferometry. While this binding affinity was relatively weak (in the $\mu \mathrm{M}$ range; Figure 3 ), it is in keeping with the binding affinity of HYPE for BiP [52]. It remains to be determined whether in a physiological setting, the interaction between HYPE and aSyn could be enhanced by the presence of BiP.

Our LC-MS/MS analysis identified three sites of adenylylation on aSyn - T33, T54 and T75. We attempted to validate these sites in vitro using site directed mutants of each of these sites. However, these adenylylation site-mutants of aSyn could still be modified in an in vitro adenylylation reaction (data not shown), likely because of secondary-site modifications, as is often seen with Fic and other enzymes [6] [7]. Interestingly, all the adenylylation sites on aSyn that we identified localized to domains of aSyn that are critical for membrane association and have also been reported as sites of O-GlcAcylation [53]. Primarily an intrinsically disordered protein, binding to the phospholipid membrane induces a structural rearrangement causing aSyn to form a helical structure [42-45]. A partial disruption of aSyn-membrane interactions contributes to aSyn aggregation at the membrane surface, a process that is apparently coupled to vesicle disruption [34, 37, 42, 46, 47]. We, therefore, assessed the physiological implications of aSyn adenylylation in PD using an in vitro liposome-based assay for measuring phospholipid membrane permeability, and found that adenylylation of aSyn reduces aSyn-mediated membrane permeabilization (Figure 7). In a previous study, substitutions at threonine residues in the amphipathic helical domain, including T33, were found to disrupt aSyn membrane binding [48]. By analogy with these earlier findings, adenylylation of aSyn threonine residues could perturb aSyn-lipid interactions. This effect, as well as a potential adenylylation-mediated disruption of interactions between neighboring aSyn molecules at the membrane surface, could lead to a decrease in the protein's ability to elicit vesicle permeabilization [34].

Of note is that adenylylation led to a decrease in the thioflavin $T$ fluorescence in solutions of aSyn incubated under fibrillation conditions (Figure 5). We also observed a change in the fibril morphology of adenylylated aSyn (Figure 6). Typical aSyn fibrils display a bundling pattern that consists of twisted fibrils $[49,50]$. We found that adenylyated aSyn fibrils existed largely as bundles of parallel fibrils (Figure 6D), suggesting (as one possibility) that addition of an AMP causes steric hindrance that prevents the fibrils from becoming closely intertwined. Our 
observation that aSyn adenylylation influences fibril morphology also implies that the difference in thioflavin $\mathrm{T}$ fluorescence shown in Figure 5 may reflect a combined effect of, in part, a difference in the quantum yield of the dye bound to the different fibril polymorphs, as well as a decrease in the extent of fibrillation of the adenylylated protein.

Collectively, our findings indicate that aSyn adenylylation interferes with aSyn fibril formation in solution and with aSyn-mediated vesicle disruption, and changes the preferred morphology of the fibrils. Evidence suggests that each of these effects could impact aSyn pathology and interfere with aSyn neurotoxicity $[49,50]$. Thus, physiologically, we propose that adenylylation may be triggered as a protective response to cope with the formation of toxic aSyn aggregates.

As mentioned earlier, HYPE plays a key role in regulating the cell's response to ER stress, and aSyn aggregation is also known to trigger ER stress and the UPR pathway via its interactions with BiP. An important question remains whether HYPE-mediated adenylylation of aSyn occurs in response to ER stress or whether adenylylation of aSyn is independent of UPR activation. Preliminary quantitative RT-PCR analysis of cDNA isolated from human control and PD patient samples revealed that HYPE's transcriptional levels did not correlate with other UPR associated transcripts (data not shown). Experiments are underway to determine whether HYPE, which we have shown to bind misfolded proteins directly in vitro, may bind and adenylylate aSyn independently of the UPR response.

In summary, we have identified aSyn as a novel target for the human Fic protein, HYPE. In doing so, we have uncovered a potential neuroprotective role for Fic-mediated adenylylation in PD that may function concurrently with HYPE's effects on chaperone activity. It remains to be determined whether the interaction of HYPE with aSyn is connected to ER stress. However, our discovery of a new, physiologically relevant target for HYPE provides significant insights for catalysis and substrate recognition, and opens a new line of research for combatting PD. Given the central role HYPE plays in cell fate, targeting HYPE as opposed to aSyn directly allows a chance to preempt the effects of aSyn aggregation and protect the cell from potential stressrelated damage. 


\section{LEGENDS}

Figure 1: A) Schematic representation of human HYPE. HYPE's Fic motif with the catalytic H363 and its a-inh domain (yellow box) with E234 are indicated. SS/TM, signal sequence/transmembrane domain. TPR, tetratricopeptide domain. Fic, filamentation induced by cAMP domain. B) Schematic representation of human alpha-Synuclein ( $\alpha$ Syn). Familial mutants associated with Parkinson's disease (A30P, E46K, H50Q, G51D, A53E, and A53T) are indicated. Sites of adenylylation (T33, T54, and T75) are indicated in red. aSyn's N-terminal (Nterm), central hydrophobic NAC (non-Abeta component of Alzheimer's disease amyloid), and Cterminal (C-term) domains are shown. Schematic representations for HYPE and aSyn are not to scale.

Figure 2: HYPE is expressed in adult and embryonic rat midbrain neurons. A) Top panel: Coronal section of a rat brain probed with antibody to HYPE and visualized with peroxide-based secondary staining shows strong HYPE expression in the substantia nigra and hippocampus. Scale bar $=2 \mathrm{~mm}$. Bottom panels: Higher magnification views of HYPE staining in the nigral region. Scale bars in the red and blue boxes represent $100 \mu \mathrm{m}$ and $50 \mu \mathrm{m}$, respectively. B) Immunofluorescence imaging of primary midbrain cultures from E17 rat embryos shows colocalization of HYPE (green, top panel) or another ER lumenal protein, BiP (green, bottom panel), with the neuronal marker MAP2 (red). HYPE shows a preferential neuronal expression, whereas BiP is expressed highly in both neurons and surrounding glial cells. Both HYPE and BiP selectively localize to the cell body in neurons. Scale bar $=50 \mu \mathrm{m}$. C) Immunofluorescence showing co-localization of HYPE (red) with the dopaminergic neuronal marker TH (tyrosine hydroxylase; green).

Figure 3: HYPE binds to and adenylylates aSyn. A) Steady state binding affinity determination for E234G/H363A-HYPE incubated with His6-tagged aSyn immobilized on HIS1K sensors were obtained by Biolayer Interferometry. B) In vitro adenylylation assay with purified HYPE and aSyn using $\alpha^{32}$ P-ATP as a nucleotide source showing adenylylation of aSyn by enzymatically active E234G-HYPE but not by its catalytically dead mutant E234G/H363A-HYPE.

Figure 4: Fragmentation of AMP-modified peptides. Figures $A, B$, and $C$ represent the fragmentation of AMP-modified peptides in MS2 (top) and MS3 (bottom) of the neutral loss fragment of AMP. A) MS2 and MS3 fragmentation of peptide $T^{33} A M P K E G V L Y V G S K$. B) MS2 and MS3 fragmentation of peptide TKEGVVHGVAT ${ }^{54}$ AMPVAEK. C) MS2 fragmentation of $\mathrm{m} / \mathrm{z}$ 755.36 of peptide TKEQVTNVGGAVVTGVT ${ }^{75}$ AMPAVAQK. Annotated are fragments that lose AMP, adenosine or adenine, either from $1+$ ions or $2+$ ions (2+). The most abundant ion (-AMP) was subsequently isolated and fragmented again to produce fragments to identify the peptide. B and $y$ ions are annotated as well as their respective sequences/amino acids.

Figure 5: HYPE-mediated adenylylation of aSyn apparently inhibits fibril elongation without altering the lag time. A) Representative kinetic traces of fibrillation assays conducted with purified aSyn adenylylated in the presence of E234G HYPE (green line) or left unmodified as aSyn alone (blue line) show a significant reduction in the yield of ThT fluorescence potentially reflecting inhibition of fibril elongation - as a result of $\alpha$ Syn adenylylation. Control samples consist of aSyn incubated in the presence of WT HYPE (red line) or catalytically dead E234G/H363A HYPE (purple line) prior to fibrillation. B) Quantification of endpoint ThT signal intensities obtained from $\mathrm{N}=3$ fibrillation assays. Asterisk * indicates a $p$ value $=0.02$. C) Endpoint fibril samples from (A) were ultracentrifuged, and soluble aSyn in the supernatant (S) fraction and insoluble/aggregated aSyn in the pellet $(P)$ fraction were analyzed by SDS-PAGE. D) Quantification of the bands corresponding to monomeric aSyn in the pellet fractions of the 
gel shown in (C). Adenylylation of aSyn following incubation with E234G-HYPE results in a decrease $(74 \%$ vs. $100 \%)$ in the amount of aSyn seen in the pellet fraction, suggesting that more aSyn is soluble/not aggregated. E) Normalized ThT values indicate that the lag time of fibrillation is unaltered by HYPE-mediated adenylylation, as the curves for WT aSyn and aSyn incubated with various HYPE samples overlap. In contrast, control samples of mouse WT aSyn (dark blue line) or human A53T aSyn mutant (orange line) exhibit a shorter lag time, indicating a much faster rate of nucleation.

Figure 6: Adenylylation alters the morphology of aSyn fibrils. Transmission electron microscopy images of fibrils formed by human WT aSyn. The protein was untreated (A) or incubated with WT HYPE (B), its catalytically inactive E234G/H363A mutant, (C) or the constitutively adenylylation competent E234G mutant (D). Fibrils formed by aSyn largely consist of bundles of fibril pairs that are intertwined, with each twist occurring at regular pitch lengths (red arrows). However, significant levels of fibrils formed by adenylylated aSyn (incubated with E234G) do not display this twist and are instead structured as parallel fibril pairs (yellow arrows). Magnified images of fibrils showing one representative twist $(A-C)$ or one parallel fibril polymorph (D) are shown beside each image. Scale $200 \mathrm{~nm}$.

Figure 7: Adenylylation reduces aSyn's ability to disrupt membranes. Calcein-loaded PG: PC (1:1) SUVs were incubated with adenylylated or non-adenylylated (control) aSyn and examined for an increase in calcein fluorescence (excitation and emission wavelengths, $485 \mathrm{~nm}$ and $515 \mathrm{~nm}$ ). The data are presented as relative membrane permeabilization after $12 \mathrm{~h}$, normalized with respect to non-adenylylated WT aSyn control. Mean \pm SEM, $n=4$ (each with 3 technical replicates). 


\section{Supplemental Legends:}

Figure S1: Additional coronal sections of a rat brain ( $n=2$ and $n=3$ ) probed with antibody to HYPE and visualized with peroxide-based secondary staining. HYPE-specific staining of the substantia nigra is shown at 10X and 20X magnification. Scale bars represent $100 \mu \mathrm{m}$ and 50 $\mu \mathrm{m}$, respectively

Figure S2: Immunofluorescence images of a rat primary midbrain culture stained for HYPE (green, left panel) or $\mathrm{BiP}$ (red, middle panel). The right panel consists of a merged image showing HYPE and BiP immunoreactivity as well as DAPI-stained nuclei. HYPE is preferentially expressed in neurons, whereas BiP staining reflects glial and neuronal expression. Both proteins appear to be preferentially expressed in neuronal cell bodies. Scale bar represents 100 $\mu \mathrm{m}$.

Figure S3: Kinetic traces of a fibrillation assay conducted with purified human WT aSyn adenylylated in the presence of E234G HYPE (green line) or left unmodified as aSyn alone (blue line) show a significant reduction in the yield of ThT fluorescence - potentially reflecting inhibition of fibril elongation - as a result of $\alpha$ Syn adenylylation. Control samples consist of $\alpha$ Syn incubated in the presence of WT-HYPE (red line) or catalytically dead E234G/H363A HYPE (purple line) prior to fibrillation. The background fluorescence for the ThT only control against which all samples were blanked is also shown (light blue line). 
KEYWORDS: Fic / Adenylylation / AMPylation / Parkinson's Disease / aSynuclein

\author{
ABBREVIATIONS: \\ AMP: Adenosine monophosphate \\ BiP: Binding immunoglobulin protein \\ BLI: Biolayer Inferometry \\ ER: Endoplasmic reticulum \\ FIC: Filamentation induced by cAMP \\ GFP: Green fluorescence protein \\ GS-AT: Glutamine synthase adenylyltransferase \\ HYPE: Huntingtin yeast interacting protein $E$ \\ LC-MS: Liquid chromatography - mass spectrometry \\ MAP2: Microtubule associated protein 2 \\ NAC: Non-amyloid-beta component of Alzheimer's disease \\ PD: Parkinson's Disease \\ PTM: Post-translational modification \\ SUV: Small unilamellar vesicles \\ TEM: Transmission electron microscopy \\ TH: Tyrosine hydroxylase \\ UPR: Unfolded protein response \\ WT: wild type
}




\section{MATERIALS AND METHODS}

\section{Cloning and plasmids}

For bacterial expression, WT aSyn/pT7-7 plasmid was procured from Addgene (www.addgene.com; plasmid \#36046). This vector was also used as a backbone for cloning $\mathrm{His}_{6}$-tagged WT-aSyn by PCR amplification.

\section{Protein expression and purification}

Untagged human WT aSyn, its A53T variant, and mouse WT aSyn purified as previously described [34]. Oligomeric species of untagged aSyn were separated from low molecular weight, mostly monomeric, species by passing the protein over a $100 \mathrm{kDa}$ cut-off filter. His ${ }_{6}$-tagged aSyn was expressed in E. coli BL21-DE3 (Stratagene) in LB medium containing $100 \mu \mathrm{g} / \mathrm{ml}$ of ampicillin to a density of $A_{600}=0.6$. Protein expression was induced for 3 hours at $37^{\circ} \mathrm{C}$ with 0.4 mM IPTG. Cells were lysed in lysis buffer (50 mM HEPES pH 8.0, $250 \mathrm{mM} \mathrm{NaCl}, 5 \mathrm{mM}$ imidazole, $10 \%(\mathrm{v} / \mathrm{v})$ glycerol) containing $1 \mathrm{mM}$ PMSF and purified using a cobalt resin. Resin was washed with wash buffer (50 mM HEPES pH 8.0, $250 \mathrm{mM} \mathrm{NaCl}, 40 \mathrm{mM}$ imidazole,10\% $(\mathrm{v} / \mathrm{v})$ glycerol) and aSyn-His protein was eluted from the resin using elution buffer $(50 \mathrm{mM}$ HEPES pH 8.0, $250 \mathrm{mM} \mathrm{NaCl}, 350 \mathrm{mM}$ imidazole, 10\% (v/v) glycerol) and further purified by ion exchange chromatography using $100 \mathrm{mM}$ Tris- $\mathrm{HCl} \mathrm{pH} 7.5$ with a salt gradient from $10 \mathrm{mM}-1 \mathrm{M}$ $\mathrm{NaCl}$. Fractions containing aSyn were purified by size exclusion chromatography in buffer containing $100 \mathrm{mM}$ Tris- $\mathrm{HCl} \mathrm{pH} 7.5,100 \mathrm{mM} \mathrm{NaCl}$ to separate monomer and the void-volume oligomer. Protein concentrations were measured using the Bradford method. Purity was determined by SDS-PAGE and proteins were stored at $-80^{\circ} \mathrm{C}$.

Recombinant HYPE and its mutants were purified as previously described [7]. Specifically, wild type and HYPE mutant proteins were expressed in E. coli BL21-DE3-RILP (Stratagene) in LB medium containing $50 \mu \mathrm{g} / \mathrm{ml}$ of kanamycin (pSMT3) to a density of $A_{600}=0.6$. Protein expression was induced for $12-16$ hours at $18^{\circ} \mathrm{C}$ with $0.4 \mathrm{mM}$ IPTG. Cells were lysed in lysis buffer (50 mM HEPES pH 8.0, $250 \mathrm{mM} \mathrm{NaCl}, 5 \mathrm{mM}$ imidazole) containing $1 \mathrm{mM}$ PMSF protease inhibitor and purified using a cobalt resin. Resin was washed with wash buffer (50 mM HEPES pH 8.0, $250 \mathrm{mM} \mathrm{NaCl}, 20 \mathrm{mM}$ imidazole). His ${ }_{6}-\mathrm{SUMO}$ tag was cleaved by incubating proteins with ULP1 at $4^{\circ} \mathrm{C}$. The protein mixture was diluted in wash buffer without imidazole and re-applied to a cobalt column, and flow through containing HYPE was further purified by ion exchange chromatography using $100 \mathrm{mM}$ Tris- $\mathrm{HCl} \mathrm{pH} 7.5$ with a salt gradient from $10 \mathrm{mM}-1$ $\mathrm{M} \mathrm{NaCl}$. Fractions containing HYPE were purified by size exclusion chromatography in buffer containing $100 \mathrm{mM}$ Tris- $\mathrm{HCl} \mathrm{pH}$ 7.5, $100 \mathrm{mM} \mathrm{NaCl}$. Protein concentrations were measured using the Bradford method. Purity was determined by SDS-PAGE and proteins were stored at $80^{\circ} \mathrm{C}$.

\section{Biolayer interferometry}

The binding kinetic assays were performed on an Octet Red 384 instrument (ForteBio, Menlo Park, CA) with the Biolayer Interferometry (BLI) technique. The assays were carried out using Anti-pentaHis (HIS1K) biosensors (ForteBio, Menlo Park, CA) at $30^{\circ} \mathrm{C}$ with the plate shaking speed at $1000 \mathrm{rpm}$. HIS1K sensors were dipped into the wells of $20 \mathrm{mM} \mathrm{His}_{6}$-aSyn in HEPES buffer (50 mM HEPES, $150 \mathrm{mM} \mathrm{NaCl}, \mathrm{pH} 7.5$ ) for 2 minutes to load aSyn on the sensors, followed by incubation in buffer for a minute to build a baseline. Next, the sensors were dipped into wells of N-terminally His tagged HYPE in HEPES Buffer at a concentration ranging from 1 $\mu \mathrm{M}$ to $150 \mu \mathrm{M}$ for 2 minutes to obtain association curves. The sensors were then dipped back to the baseline HEPES buffer wells for 3 minutes to obtain dissociation curves. The data were analyzed by the ForteBio data analysis software v.9.0.0.12. The binding constants $(\mathrm{Kd})$ and kinetic parameters were determined by globally fitting data to the $1: 1$ fitting model. 


\section{In vitro adenylylation assays}

$0.5 \mu \mathrm{g}$ of E234G or E234G/H363A HYPE (aa 102-458) protein was incubated either alone or with $5 \mu \mathrm{g}$ of untagged aSyn monomer or oligomer in an adenylylation reaction containing $5 \mathrm{mM}$ HEPES pH 7.5, $1 \mathrm{mM}$ manganese chloride tetrahydrate, $0.5 \mathrm{mM}$ EDTA and $0.01 \mathrm{mCi} \mathrm{a}^{32} \mathrm{P}$-ATP for 15 minutes. Reactions were stopped with SDS PAGE loading buffer, separated on $4-20 \%$ polyacrylamide gels and visualized by autoradiography.

\section{Mammalian cell culture assays}

HEK293T cells were cultured in Dulbecco's Modified Eagle Medium (Sigma-Aldrich) supplemented with $5 \%(\mathrm{v} / \mathrm{v})$ fetal bovine serum (Life Technology) and $100 \mathrm{~g} / \mathrm{ml}$ penicillin/streptomycin (Sigma-Aldrich) at $37^{\circ} \mathrm{C}$ with $5 \% \mathrm{CO}_{2}$. Fugene $\mathrm{HD}$ (Promega) was used for transfections according to the manufacturer's directions. Cells were transfected for $30 \mathrm{hrs}$. Primary midbrain cultures were prepared and treated as previously described [34].

\section{Mass spectrometry}

For in-gel protein digestion, gel bands were cut out, destained, reduced with 5mM DTT and alkylated with $10 \mathrm{mM}$ iodoacetamide and digested with either trypsin at $37 \mathrm{C}$ or chymotrypsin at room temperature essentially as described by Shevchenko et al. [51] with minor modifications. For LC-MS/MS analysis, the dried peptide mix was reconstituted in a solution of $2 \%$ formic acid (FA) for MS analysis. Peptides were loaded with the autosampler directly onto a $2 \mathrm{~cm}$ C18 PepMap pre-column (Thermo Scientific, San Jose, CA) which was attached to a $50 \mathrm{~cm}$ EASY-Spray C18 column (Thermo Scientific). Peptides were eluted from the column using a Dionex Ultimate 3000 Nano LC system with a 1.5 min gradient from $2 \%$ buffer B to $5 \%$ buffer B ( $100 \%$ acetonitrile, $0.1 \%$ formic acid), followed by a 15 min gradient to $20 \%$ of $B$, followed by a 2 min gradient to $30 \%$ of $\mathrm{B}$. The gradient was switched from $30 \%$ to $85 \%$ buffer B over 1 min and held constant for 1 min. Finally, the gradient was changed from $85 \%$ buffer B to $98 \%$ buffer A (100\% water, $0.1 \%$ formic acid) over $1 \mathrm{~min}$, and then held constant at $98 \%$ t buffer $\mathrm{A}$ for 15 more minutes. The application of a $2.0 \mathrm{kV}$ distal voltage electrosprayed the eluting peptides directly into the Thermo Fusion Tribrid mass spectrometer equipped with an EASY-Spray source (Thermo Scientific). Mass spectrometer-scanning functions and HPLC gradients were controlled by the Xcalibur data system (Thermo Scientific). Initial experiments were performed to identify AMP modified peptides by running each digest separately with a data dependent method to acquire as many MS/MS in a 3 second span. This data was analyzed for the presence of AMP modification with either inspect (proteomics.ucsd.edu) or Thermo Proteome Discoverer 2.1 and looking for a modification of 329.05252 on either tyrosine, threonine or serine or by manually looking at MS/MS scans and looking for the specific signal for AMP (m/z 348.08). Three MS/MS scans were identified that contained AMP, however, the most abundant peak was identified as the neutral loss of AMP from the parent mass. Therefore an analysis was conducted that targeted these neutral loss ions for an additional MS3 scan to identify the peptide sequence and position of the AMP modification.

\section{Immunocytochemistry and immunohistochemistry (IHC)}

Primary midbrain cultures were fixed with $4 \%(\mathrm{w} / \mathrm{v})$ paraformaldehyde and blocked with 1X PBS containing 10\% (v/v) Fetal Bovine Serum (FBS), 1\% (w/v) BSA and 0.3\% (v/v) Triton X100 for 1 hour. This was followed by treatment with primary antibodies - mouse anti-HYPE (Abcam cat\# ab67163, 1:100), chicken anti-MAP2 (Encor cat\# CPCA-MAP2, 1:2000) or rabbit anti-TH (Phosphosolutions cat\# 2025-THRAB, 1:1000) diluted in 1X PBS containing 1\% (w/v) $\mathrm{BSA}$ and incubated overnight at $4^{\circ} \mathrm{C}$. The cells were washed and incubated with secondary antibodies Alexa anti-chicken 594, Alexa anti-rabbit 488 or Alexa anti-mouse 594 for 1 hour at 
room temperature, washed, and imaged using the CYTATION 3 Cell Imaging Reader or Nikon Ti confocal microscope

Rat brain sections $(40 \mu \mathrm{m})$ were obtained using a microtome and stored in cryoprotectant solution at $-20^{\circ} \mathrm{C}$. On the day of the experiment, sections were transferred to a separate plate and washed six times with PBS. All incubations were conducted while shaking at $100 \mathrm{rpm}$. The sections were blocked with 10\% Normal Donkey Serum (NDS) in 0.3\% PBSTriton for 90 minutes, treated with mouse primary antibody for HYPE (Abcam cat\# ab67163, $1: 100$ ) diluted in PBS containing $1 \%$ NDS and $0.3 \%$ Triton for 24 hours at $4^{\circ} \mathrm{C}$. The following day, the sections were washed three times with PBS, incubated with biotinylated secondary antibody anti-mouse (Jackson ImmunoResearch, cat\# 715-065-151, 1:200) for 90 minutes at room temperature, washed with PBS and incubated in the premade ABC solution from VECTASTAIN Elite ABC HRP Kit (Vector Laboratories, cat\# PK-7200) for 60 minutes. A solution of DAB (diaminobenzidine) and $\mathrm{H}_{2} \mathrm{O}_{2}$ was prepared using the DAB Peroxidase Substrate Kit (Vector Laboratories, cat\# SK-4100). The sections were were then treated with the DAB solution until a brown color developed, washed to remove non-specific staining and transferred to a slide. The tissues were allowed to dry overnight and were dehydrated using a series of solutions of increasing ethanol concentrations (70-100\%), followed by treatment with Histoclear, mounted using Histomount and dried overnight before microscopy using the Olympus BX53 light microscope.

\section{aSyn fibrillation}

Lyophilized untagged aSyn was dissolved in PBS ( $\mathrm{pH} 7.4$ ) with $0.02 \%[\mathrm{w} / \mathrm{v}] \mathrm{NaN}_{3}$, and dialyzed against the same buffer $\left(24 \mathrm{~h}\right.$ at $\left.4^{\circ} \mathrm{C}\right)$ to remove excess salt. The solution was filtered by successive centrifugation steps through a $0.22 \mu \mathrm{m}$ spin filter and a $100 \mathrm{kDa}$ centrifugal filter to isolate low molecular weight (mostly monomeric) protein. This low molecular weight $\alpha$ Syn was incubated in PBS for an hour with or without HYPE as outlined above. Fibrillation solutions of aSyn were prepared by diluting the adenylation reaction mixture (or blank) to a final concentration of $35 \mu \mathrm{M}$ in the wells of a 96-well plate. To determine the extent of aSyn fibrillation, thioflavin $\mathrm{T}$ (ThioT, final concentration, $20 \mu \mathrm{M}$ ) was added to the fibrillation solutions, which were incubated at $37^{\circ} \mathrm{C}$ with shaking at the 'normal' setting in a Spectra Fluor Plus or Genios plate reader (Tecan, Upsala, Sweden). HYPE was not removed from the fibrillation mixture as it did not interfere with fibrillation or show increased ThioT fluorescence on its own. A nylon ball was added to avoid inhomogeneous mixing of samples. ThioT fluorescence was measured with excitation at $440 \mathrm{~nm}$ and emission at $490 \mathrm{~nm}$. Mean ThioT fluorescence data determined from 2-4 technical replicates were normalized using the following equation:

$$
\text { normalized fluorescence }=\frac{\mathrm{Ft}-\mathrm{Fmin}}{\text { Fmax }- \text { Fmin }}
$$

where $F_{t}$ is the ThioT fluorescence emission intensity at time $t$, and $F_{\min }$ and $F_{\max }$ are the minimum and maximum fluorescence intensities during the incubation, respectively.

\section{Synthetic vesicle disruption assay and on-bead adenylylation}

Calcein loaded Small unilamellar vesicles (SUVs) (diameter $\sim 90 \mathrm{~nm}$ ) were prepared as described previously [37]. We opted to examine SUVs composed of egg PG and egg PC (1:1 $\mathrm{mol} / \mathrm{mol}$ ) because they contain anionic lipids necessary for aSyn membrane interactions and this lipid composition is compatible with producing stable $90 \mathrm{~nm}$ vesicles. Egg PG and egg PC suspended in chloroform were mixed in a round bottom flask. The chloroform was evaporated under a nitrogen stream and further dried under vacuum for $1 \mathrm{~h}$. Dried lipids were suspended in a solution of calcein $(170 \mathrm{mM})$ prepared in water using $\mathrm{NaOH}$ to adjust the $\mathrm{pH}$ to 7.4 . The final osmolality was $280 \mathrm{mOsm} / \mathrm{kg}$. The liposomes were sized by extruding the suspension through a 
$50 \mathrm{~nm}$ pore size Whatman membrane. Calcein-containing vesicles were isolated from free calcein via gel filtration through a Sephadex G-50 column pre-equilibrated with PBS, pH 7.4, $0.02 \%(\mathrm{w} / \mathrm{v}) \mathrm{NaN} 3(280 \mathrm{mOsm} / \mathrm{kg})$. Fractions containing isolated vesicles were pooled and stored at $4{ }^{\circ} \mathrm{C}$ until use. Calcein-loaded vesicles were found to be very stable, with no spontaneous dye leakage observed over several weeks. The size of the vesicles was confirmed by dynamic light scattering.

To avoid any interference of HYPE on vesicle permeabilization, the adenylation reaction was done in PBS for one hour as mentioned previously (the adenylation method need to added at the beginning) but with Ni-agarose bead bound HYPE. Untagged aSyn was removed from the beads by removing the supernatant after centrifugation of the samples. To remove any nonspecifically bound aSyn from the beads, the sample was washed with small volumes of PBS and pooled with the supernatant as mentioned above. aSyn concentration was calculated with BCA assay and absorbance measurement at 280nm.

For membrane disruption experiments, monomeric aSyn variants (40 $\mu \mathrm{M}$, unmodified and modified) were incubated with calcein-loaded PG:PC SUVs (protein-to-lipid ratio, 1:20 $\mathrm{mol} / \mathrm{mol}$ ) at $37^{\circ} \mathrm{C}$ in a total volume of $40 \mu \mathrm{L}$. Samples were analyzed with a TECAN fluorescence plate reader (excitation wavelength, $485 \mathrm{~nm}$; emission wavelengths, $530 \mathrm{~nm}$ ). To determine the maximum dye release, vesicles were lysed by adding $3 \mu \mathrm{L}$ of $1 \%(\mathrm{v} / \mathrm{v})$ Triton $\mathrm{X}$ 100. The percentage leakage at time t was determined using the following equation:

$$
\text { percentage leakage }=\frac{\mathrm{It}-\mathrm{I} 0}{\mathrm{Imax}-\mathrm{I} 0} \times 100 \%
$$

where It is the fluorescence emission intensity at time $t, I_{0}$ is the intensity at time 0 , and Imax is the maximal intensity determined after detergent lysis of the vesicles. aSyn-mediated leakage reached a plateau after $12-16 \mathrm{~h}$, and the plateau value was used to compare the leakage among the HYPE-modified and unmodified samples.

\section{Western blot analysis of fibril samples}

A $150 \mu \mathrm{L}$ aliquot of the fibrillation sample was centrifuged for $60 \mathrm{~min}$ at $100,000 \times \mathrm{g}$ (Beckman Coulter Optima MAX-XP) to precipitate the fibrils. The supernatant was collected to analyze the residual monomeric content. The fibril pellet was dissolved in $150 \mu \mathrm{L}$ PBS and vortexed gently and used for analysis. Appropriate amounts of the samples were mixed with Laemmli sample buffer and denatured proteins were separated via SDS-PAGE on a $12 \%(\mathrm{w} / \mathrm{v})$ polyacrylamide gel and transferred to an Immobilon-FL PVDF membrane (pore size, $0.45 \mu \mathrm{m}$ ). The membrane was probed with mouse anti-aSyn (BD Biosciences; 1:1,500), and an antimouse AP-linked secondary antibody (Cell Signaling Technology, 1: 3,000). To visualize the bands, the membrane was exposed to ECF substrate and imaged using a Typhoon imaging system (GE Life Sciences, Pittsburgh, PA). Band intensities were quantified using ImageJ software ( $\mathrm{NIH}$, Bethesda, MD), and averages calculated by quantifying each band in triplicate.

\section{Transmission Electron Microscopy (TEM) analysis of aSyn amyloid-like fibrils}

The morphology of the aSyn amyloid-like fibrils was analyzed by negative stain biological TEM. $3 \mu \mathrm{L}$ of the aSyn sample solution of interest $(35 \mu \mathrm{M}$, directly from the fibrillation assay) was pipetted on a discharged carbon-coated copper TEM grid (Electron Microscopy Sciences, CF400CU; 400 mesh) and incubated for $1 \mathrm{~min}$. Subsequently, the sample was washed with deionized water carefully without letting it dry and stained with a $1 \%(w / v)$ phosphotungstic acid (PTA) solution $(3 \mu \mathrm{L})$, which was left in contact with the protein on the grid for $1 \mathrm{~min}$. The excess solution was then removed by blotting with filter paper, and the sample was imaged using an FEI Tecnai G2 20 Transmission Electron Microscope operating at $200 \mathrm{KV}$. 


\section{Acknowledgments \\ We thank the Biophysical Analysis Laboratory at Purdue University's Bindley Biosciences Center for use its Octet Red 384 for BLI analysis, and Dr. Chris Gilpin at the Purdue Electron Microscopy Core for use of the FEI Tecnai G2 20 TEM. Finally, we are grateful to members of the Mattoo and Rochet labs for their helpful discussions.}

Declaration of interest The authors declare no conflict of interest with the contents of this article.

Funding information This work was funded in part by the National Institute of General Medical Sciences of the National Institute of Health (R01GM10092), an Indiana Clinical and Translational Research Grant (CTSI-106564), and a Purdue Institute for Inflammation, Immunology, and Infectious Disease Core Start Grant (PI4D-209263) to SM; a grant from the Branfman Family Foundation to JCR; a grant from the National Institutes of Health (R01ES025750) to JRC; grants from the Purdue Research Foundation (PRF-209104) and the Cancer Prevention Internship Program to AS, and Eli Lilly-Stark Neuroscience Research Institute-CTSI pre-doctoral fellowship to SD.

\section{Author contributions}

S.M. conceived the study. S.M., J-C.R., A.S., and S.D. designed the study. A.S., S.D., A.C., A.C., and B.G.W. conducted the experiments. D.Y. and P.M. provided initial samples of purified aSyn and followed protocols developed by J.R.C. for handling rat brain samples. R.S. helped with TEM experiments. A.K. conducted mass spectrometric analyses. S.M. and J-C.R. supervised the study. S.M. and A.S. wrote the manuscript. All authors revised and agreed to the final version of the manuscript. 


\section{REFERENCES:}

1. Casey AK, Orth K. Enzymes Involved in AMPylation and deAMPylation. Chem Rev. 2018;118:1199-215.

2. Worby CA, Mattoo S, Kruger RP, Corbeil LB, Koller A, Mendez JC, et al. The fic domain: regulation of cell signaling by adenylylation. Mol Cell. 2009;34:93-103.

3. Engel P, Goepfert A, Stanger FV, Harms A, Schmidt A, Schirmer T, et al. Adenylylation control by intra- or intermolecular active-site obstruction in Fic proteins. Nature. 2012;482:107-10.

4. Mattoo S, Durrant E, Chen MJ, Xiao J, Lazar CS, Manning G, et al. Comparative analysis of Histophilus somni immunoglobulin-binding protein $A$ (IbpA) with other fic domain-containing enzymes reveals differences in substrate and nucleotide specificities. J Biol Chem. 2011;286:32834-42.

5. Zekarias B, Mattoo S, Worby C, Lehmann J, Rosenbusch RF, Corbeil LB. Histophilus somni IbpA DR2/Fic in virulence and immunoprotection at the natural host alveolar epithelial barrier. Infect Immun. 2010;78:1850-8.

6. Xiao J, Worby CA, Mattoo S, Sankaran B, Dixon JE. Structural basis of Fic-mediated adenylylation. Nat Struct Mol Biol. 2010;17:1004-10.

7. Sanyal A, Chen AJ, Nakayasu ES, Lazar CS, Zbornik EA, Worby CA, et al. A novel link between Fic (filamentation induced by CAMP)-mediated adenylylation/AMPylation and the unfolded protein response. J Biol Chem. 2015;290:8482-99.

8. Ron $D$, Walter $P$. Signal integration in the endoplasmic reticulum unfolded protein response. Nat Rev Mol Cell Biol. 2007;8:519-29.

9. Kaufman RJ. Orchestrating the unfolded protein response in health and disease. $\mathrm{J}$ Clin Invest. 2002;110:1389-98.

10. Preissler S, Rato C, Perera L, Saudek V, Ron D. FICD acts bifunctionally to AMPylate and de-AMPylate the endoplasmic reticulum chaperone BiP. Nat Struct Mol Biol. 2017;24:23-9.

11. Mallamace F, Corsaro C, Mallamace D, Vasi S, Vasi C, Baglioni P, et al. Energy landscape in protein folding and unfolding. Proc Natl Acad Sci U S A. 2016;113:3159-63.

12. Hellman R, Vanhove M, Lejeune A, Stevens FJ, Hendershot LM. The in vivo association of $\mathrm{BiP}$ with newly synthesized proteins is dependent on the rate and stability of folding and not simply on the presence of sequences that can bind to BiP. $J$ Cell Biol. 1999;144:21-30.

13. Auer S, Dobson CM, Vendruscolo M. Characterization of the nucleation barriers for protein aggregation and amyloid formation. HFSP J. 2007;1:137-46.

14. Herczenik E, Gebbink MF. Molecular and cellular aspects of protein misfolding and disease. FASEB J. 2008;22:2115-33.

15. Cuenca L, Gil-Martinez AL, Cano-Fernandez L, Sanchez-Rodrigo C, Estrada C, Fernandez-Villalba E, et al. Parkinson's disease: a short story of 200 years. Histol Histopathol. 2018:18073.

16. Spillantini MG, Schmidt ML, Lee VM, Trojanowski JQ, Jakes R, Goedert M. Alphasynuclein in Lewy bodies. Nature. 1997;388:839-40.

17. Lashuel HA, Overk CR, Oueslati A, Masliah E. The many faces of alpha-synuclein: from structure and toxicity to therapeutic target. Nat Rev Neurosci. 2013;14:38-48.

18. Ma MR, Hu ZW, Zhao YF, Chen YX, Li YM. Phosphorylation induces distinct alphasynuclein strain formation. Sci Rep. 2016;6:37130.

19. Conway KA, Lee SJ, Rochet JC, Ding TT, Williamson RE, Lansbury PT, Jr. Acceleration of oligomerization, not fibrillization, is a shared property of both alpha-synuclein mutations linked to early-onset Parkinson's disease: implications for pathogenesis and therapy. Proc Natl Acad Sci U S A. 2000;97:571-6. 
20. Fusco G, Chen SW, Williamson PTF, Cascella R, Perni M, Jarvis JA, et al. Structural basis of membrane disruption and cellular toxicity by alpha-synuclein oligomers. Science. 2017;358:1440-3.

21. Karpinar DP, Balija MB, Kugler S, Opazo F, Rezaei-Ghaleh N, Wender N, et al. Prefibrillar alpha-synuclein variants with impaired beta-structure increase neurotoxicity in Parkinson's disease models. EMBO J. 2009;28:3256-68.

22. Volles MJ, Lee SJ, Rochet JC, Shtilerman MD, Ding TT, Kessler JC, et al. Vesicle permeabilization by protofibrillar alpha-synuclein: implications for the pathogenesis and treatment of Parkinson's disease. Biochemistry. 2001;40:7812-9.

23. Winner B, Jappelli R, Maji SK, Desplats PA, Boyer L, Aigner S, et al. In vivo demonstration that alpha-synuclein oligomers are toxic. Proc Natl Acad Sci U S A. 2011;108:4194-9.

24. Colla E, Jensen PH, Pletnikova O, Troncoso JC, Glabe C, Lee MK. Accumulation of toxic alpha-synuclein oligomer within endoplasmic reticulum occurs in alphasynucleinopathy in vivo. J Neurosci. 2012;32:3301-5.

25. Mercado G, Valdes P, Hetz C. An ERcentric view of Parkinson's disease. Trends Mol Med. 2013;19:165-75.

26. Ham H, Woolery AR, Tracy C, Stenesen D, Kramer H, Orth K. Unfolded protein response-regulated Drosophila Fic (dFic) protein reversibly AMPylates BiP chaperone during endoplasmic reticulum homeostasis. J Biol Chem. 2014;289:36059-69.

27. Preissler S, Rato C, Chen R, Antrobus R, Ding S, Fearnley IM, et al. AMPylation matches BiP activity to client protein load in the endoplasmic reticulum. Elife. 2015;4:e12621.

28. Preissler S, Rohland L, Yan Y, Chen R, Read RJ, Ron D. AMPylation targets the ratelimiting step of BiP's ATPase cycle for its functional inactivation. Elife. 2017;6.

29. Truttmann MC, Pincus D, Ploegh HL. Chaperone AMPylation modulates aggregation and toxicity of neurodegenerative disease-associated polypeptides. Proc Natl Acad Sci U S A. 2018;115:E5008-E17.

30. Broncel M, Serwa RA, Bunney TD, Katan M, Tate EW. Global Profiling of Huntingtinassociated protein E (HYPE)-Mediated AMPylation through a Chemical Proteomic Approach. Mol Cell Proteomics. 2016;15:715-25.

31. Truttmann MC, Cruz VE, Guo X, Engert C, Schwartz TU, Ploegh HL. The Caenorhabditis elegans Protein FIC-1 Is an AMPylase That Covalently Modifies HeatShock 70 Family Proteins, Translation Elongation Factors and Histones. PLoS Genet. 2016;12:e1006023.

32. Grassi D, Howard S, Zhou M, Diaz-Perez N, Urban NT, Guerrero-Given D, et al. Identification of a highly neurotoxic alpha-synuclein species inducing mitochondrial damage and mitophagy in Parkinson's disease. Proc Natl Acad Sci U S A. 2018;115:E2634-E43.

33. Gorbatyuk MS, Shabashvili A, Chen W, Meyers C, Sullivan LF, Salganik M, et al. Glucose regulated protein 78 diminishes alpha-synuclein neurotoxicity in a rat model of Parkinson disease. Mol Ther. 2012;20:1327-37.

34. Ysselstein D, Joshi M, Mishra V, Griggs AM, Asiago JM, McCabe GP, et al. Effects of impaired membrane interactions on alpha-synuclein aggregation and neurotoxicity. Neurobiol Dis. 2015;79:150-63.

35. Mohite GM, Kumar R, Panigrahi R, Navalkar A, Singh N, Datta D, et al. Comparison of Kinetics, Toxicity, Oligomer Formation, and Membrane Binding Capacity of alphaSynuclein Familial Mutations at the A53 Site, Including the Newly Discovered A53V Mutation. Biochemistry. 2018;57:5183-7. 
36. Li B, Ge P, Murray KA, Sheth P, Zhang M, Nair G, et al. Cryo-EM of full-length alphasynuclein reveals fibril polymorphs with a common structural kernel. Nat Commun. 2018;9:3609.

37. Ysselstein D, Dehay B, Costantino IM, McCabe GP, Frosch MP, George JM, et al. Endosulfine-alpha inhibits membrane-induced alpha-synuclein aggregation and protects against alpha-synuclein neurotoxicity. Acta Neuropathol Commun. 2017;5:3.

38. Kingdon HS, Shapiro BM, Stadtman ER. Regulation of glutamine synthetase. 8. ATP: glutamine synthetase adenylyltransferase, an enzyme that catalyzes alterations in the regulatory properties of glutamine synthetase. Proc Natl Acad Sci U S A. 1967;58:170310.

39. Sreelatha A, Yee SS, Lopez VA, Park BC, Kinch LN, Pilch S, et al. Protein AMPylation by an Evolutionarily Conserved Pseudokinase. Cell. 2018;175:809-21 e19.

40. Lewallen DM, Steckler CJ, Knuckley B, Chalmers MJ, Thompson PR. Probing adenylation: using a fluorescently labelled ATP probe to directly label and immunoprecipitate VopS substrates. Mol Biosyst. 2012;8:1701-6.

41. Trychta KA, Back S, Henderson MJ, Harvey BK. KDEL Receptors Are Differentially Regulated to Maintain the ER Proteome under Calcium Deficiency. Cell Rep. 2018;25:1829-40 e6.

42. Bodner CR, Maltsev AS, Dobson CM, Bax A. Differential phospholipid binding of alphasynuclein variants implicated in Parkinson's disease revealed by solution NMR spectroscopy. Biochemistry. 2010;49:862-71.

43. Jao CC, Der-Sarkissian A, Chen J, Langen R. Structure of membrane-bound alphasynuclein studied by site-directed spin labeling. Proc Natl Acad Sci U $S A$. 2004;101:8331-6.

44. Bussell R, Jr., Eliezer D. A structural and functional role for 11-mer repeats in alphasynuclein and other exchangeable lipid binding proteins. J Mol Biol. 2003;329:763-78.

45. Davidson WS, Jonas A, Clayton DF, George JM. Stabilization of alpha-synuclein secondary structure upon binding to synthetic membranes. J Biol Chem. 1998;273:94439.

46. Lee JH, Hong CS, Lee S, Yang JE, Park YI, Lee D, et al. Radiating amyloid fibril formation on the surface of lipid membranes through unit-assembly of oligomeric species of alpha-synuclein. PLoS One. 2012;7:e47580.

47. Reynolds NP, Soragni A, Rabe M, Verdes D, Liverani E, Handschin S, et al. Mechanism of membrane interaction and disruption by alpha-synuclein. $J$ Am Chem Soc. 2011;133:19366-75.

48. Perrin RJ, Woods WS, Clayton DF, George JM. Interaction of human alpha-Synuclein and Parkinson's disease variants with phospholipids. Structural analysis using sitedirected mutagenesis. J Biol Chem. 2000;275:34393-8.

49. Peelaerts W, Bousset L, Van der Perren A, Moskalyuk A, Pulizzi R, Giugliano M, et al. alpha-Synuclein strains cause distinct synucleinopathies after local and systemic administration. Nature. 2015;522:340-4.

50. Bousset L, Pieri L, Ruiz-Arlandis G, Gath J, Jensen PH, Habenstein B, et al. Structural and functional characterization of two alpha-synuclein strains. Nat Commun. 2013; $4: 2575$.

51. Shevchenko A, Wilm M, Vorm O, Mann M. Mass spectrometric sequencing of proteins silver-stained polyacrylamide gels. Anal Chem. 1996; 68:850-8.

52. Sanyal A, Zbornik EA, Watson BG, Christoffer C, Ma J, Kihara D. and Mattoo S, Kinetic And Structural Parameters Governing Fic-Mediated Adenylylation/AMPylation of the Hsp70 chaperone, BiP/GRP78. Biorxiv 494930; doi: https://doi.org/10.1101/494930

53. Levine PM, Galesic A, Balana AT, Mahul-Mellier A-L, Navarro MX, De Leon CA, et al. aSynculein O-GlcNAcylation alters aggregation and toxicity, revealing certain residues 
as potential inhibitors of Parkinson's disease. Proc Nat Acad Sci. 2019; doi:10.1073/pnas.1808845116

54. Sengupta R, Poderycki MJ, and Mattoo S. Cryo-APEX: An electron tomography tool for subcellular localization of membrane proteins. bioRxiv 522482; doi: https://doi.org/10.1101/522482 
A:

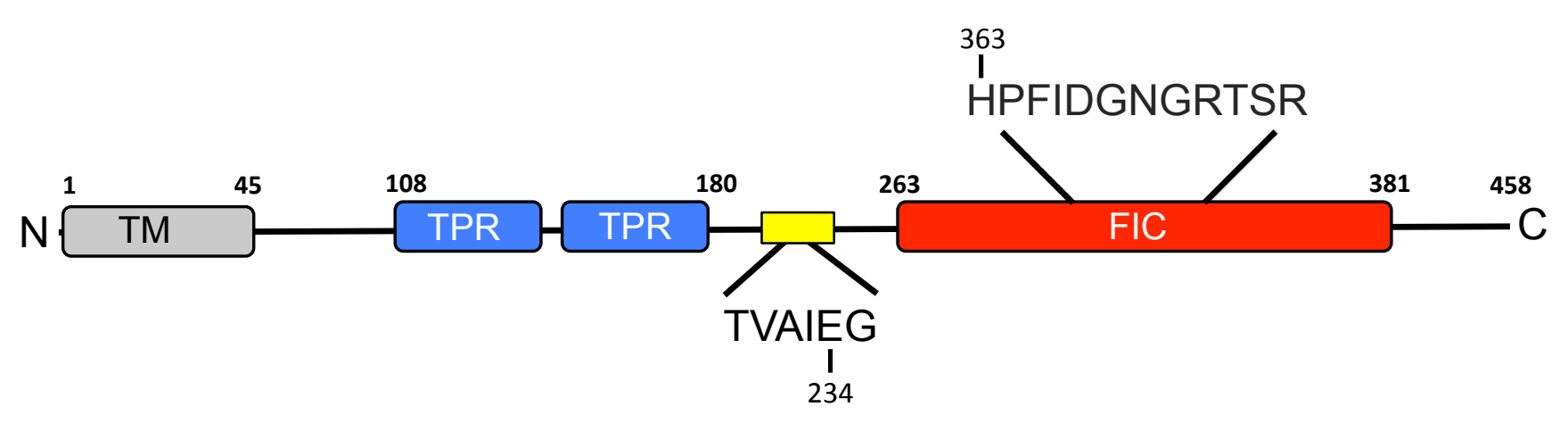

B:

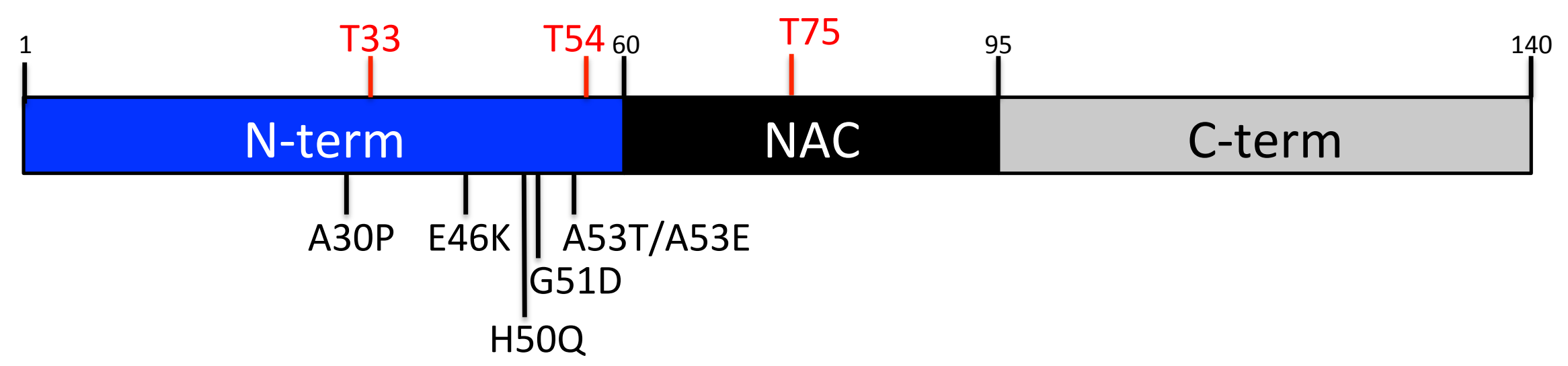




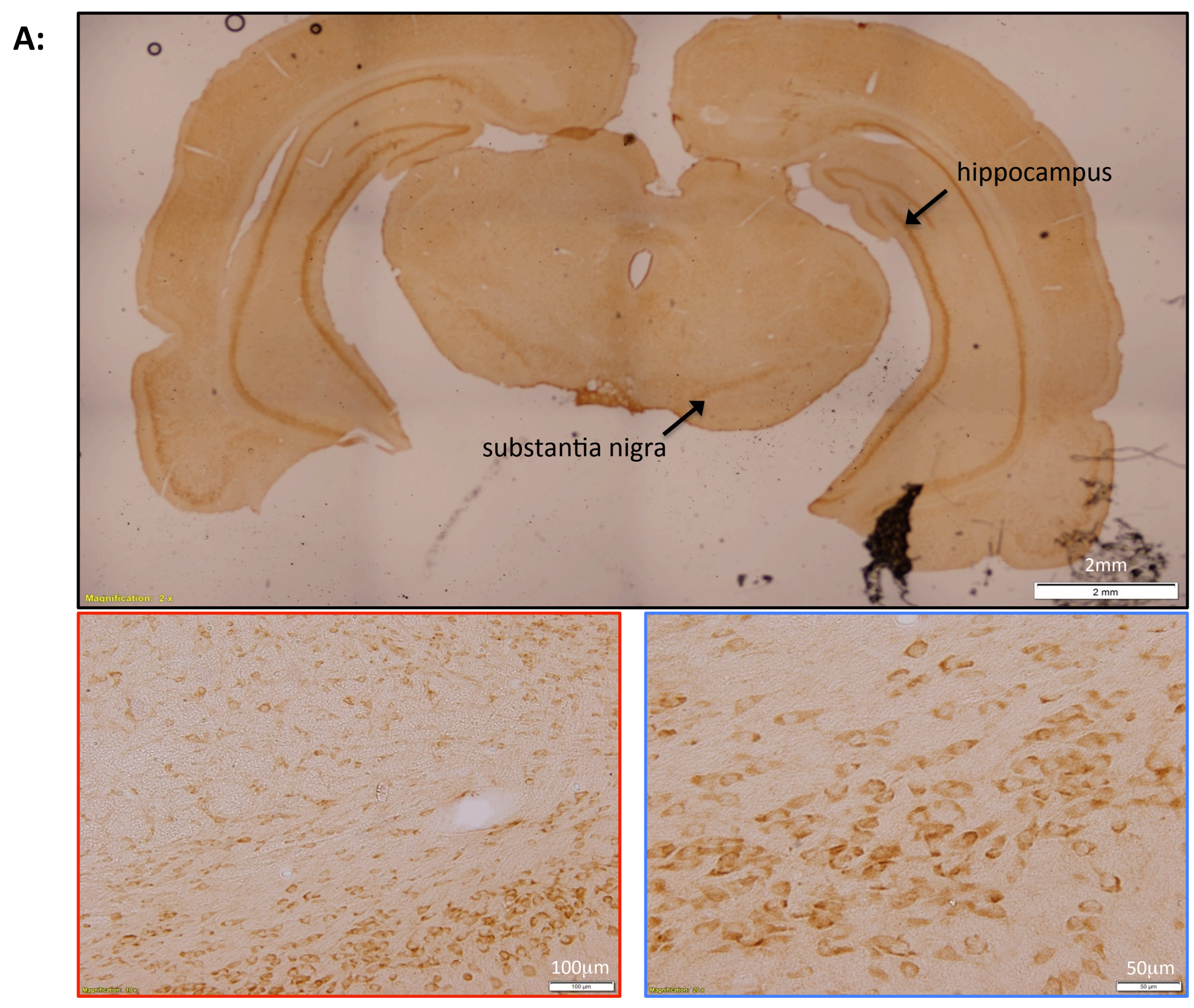


Sanyal \& Dutta et al., 2019 - Figure 2

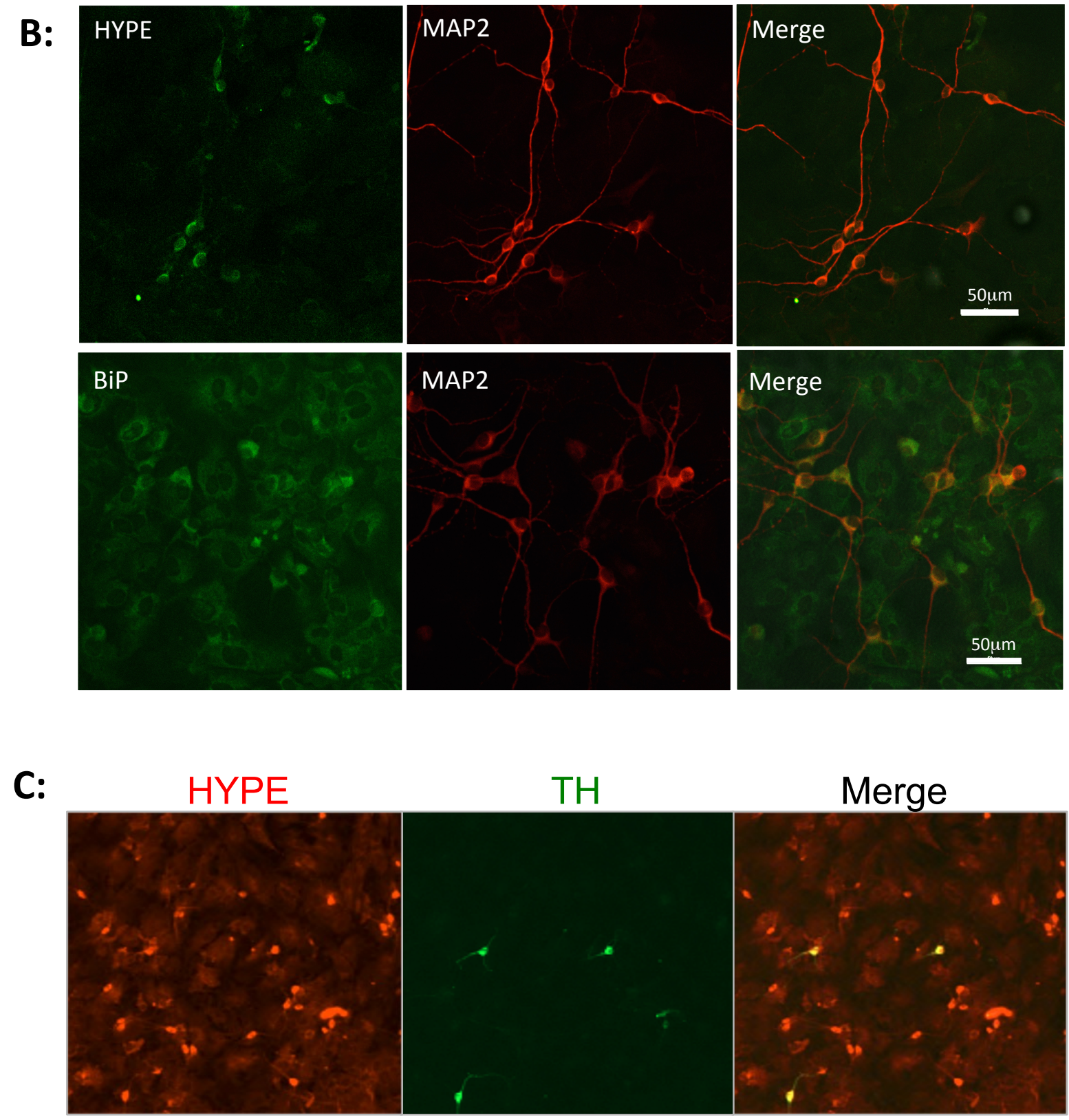


A:

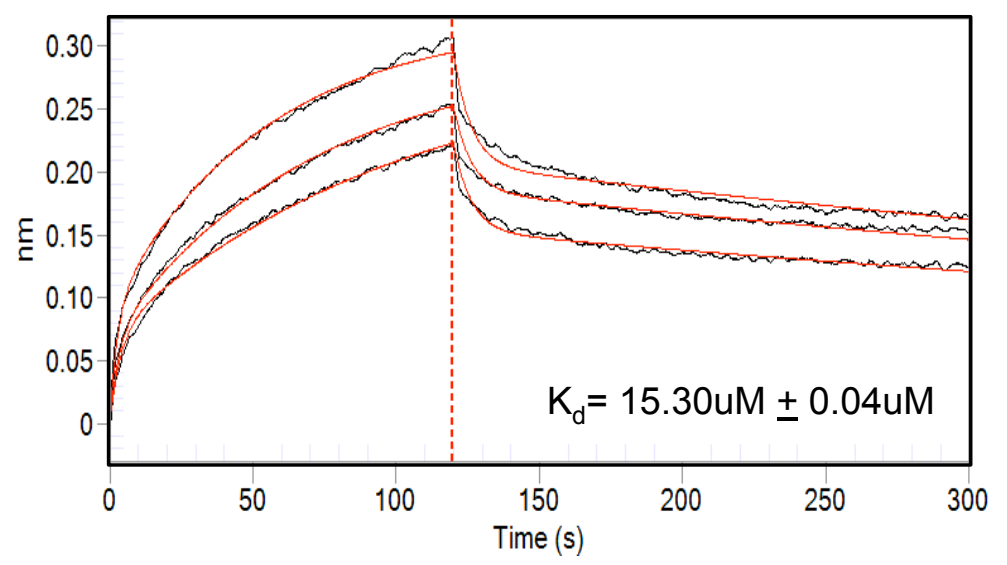

B:

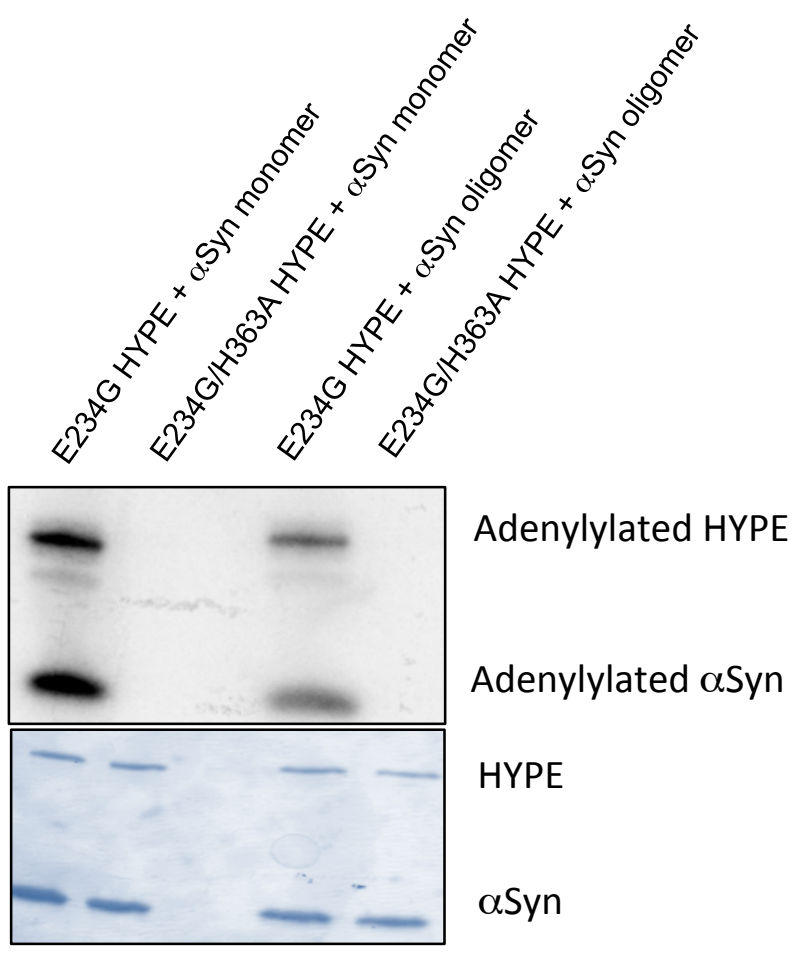


Sanyal, Dutta et al., 2019 - Figure 4A

\section{$(\mathrm{K}) T_{33}{ }^{\mathrm{AMP}} \operatorname{KEGVLYVGSK}(\mathrm{T})$}

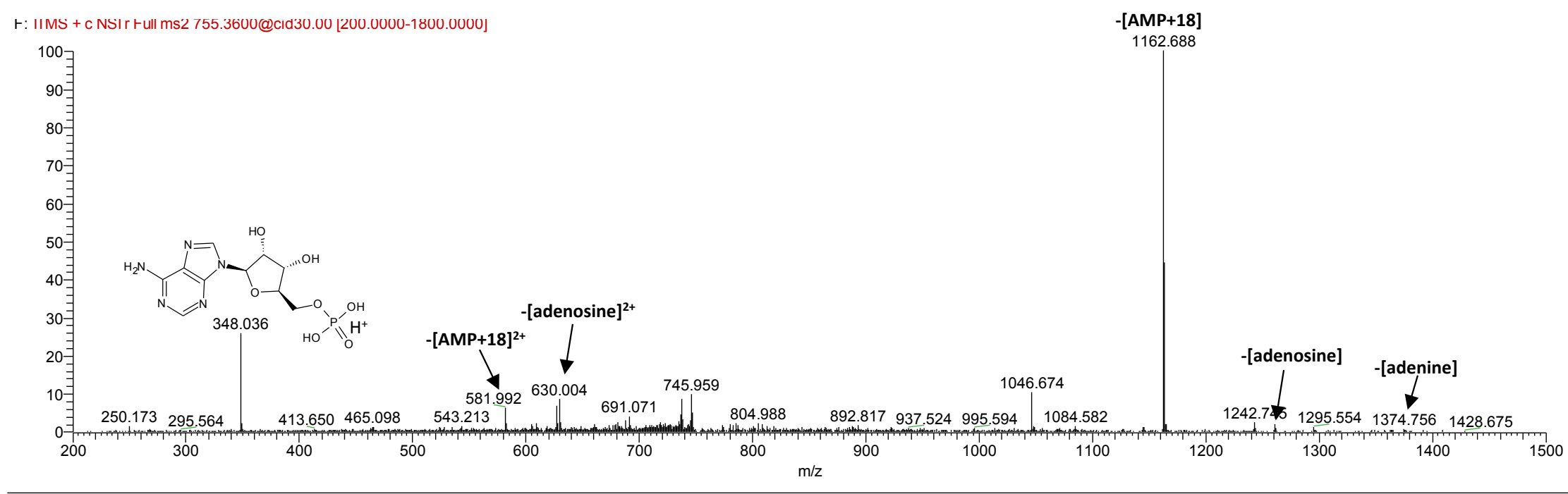

F: ITMS + c NSI r Full ms3 755.4000@cid30.00 1162.6000@cid30.00 [200.0000-2000.0000]

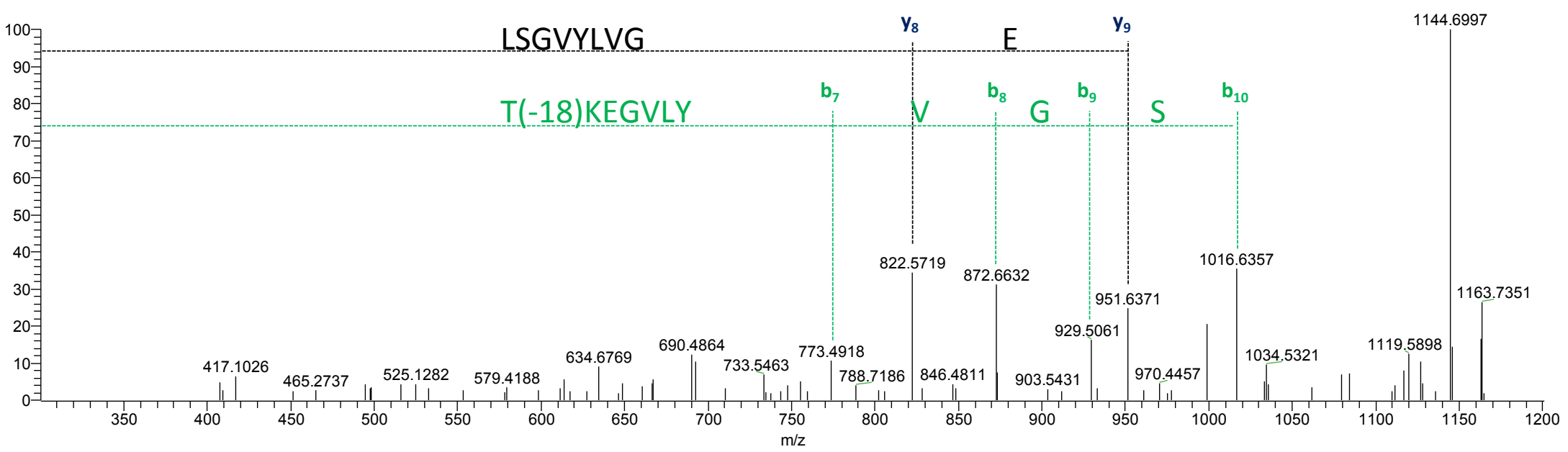


Sanyal \& Dutta et al., 2019 - Figure 4B

\section{(K)TKEGVVHGVA $T_{54}{ }^{A M P} \operatorname{VAEK}(\mathrm{T})$}

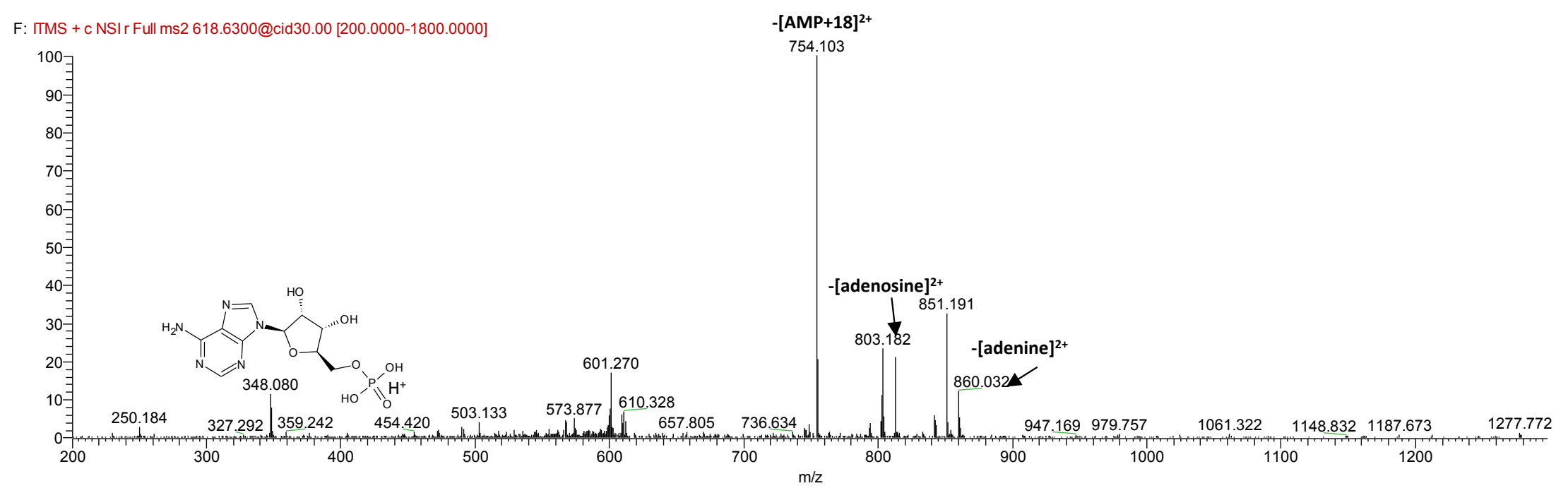

F: TMS + c NSI r Full ms3618.6000@cid30.00 754.1000@cid30.00 [200.0000-2000.0000]

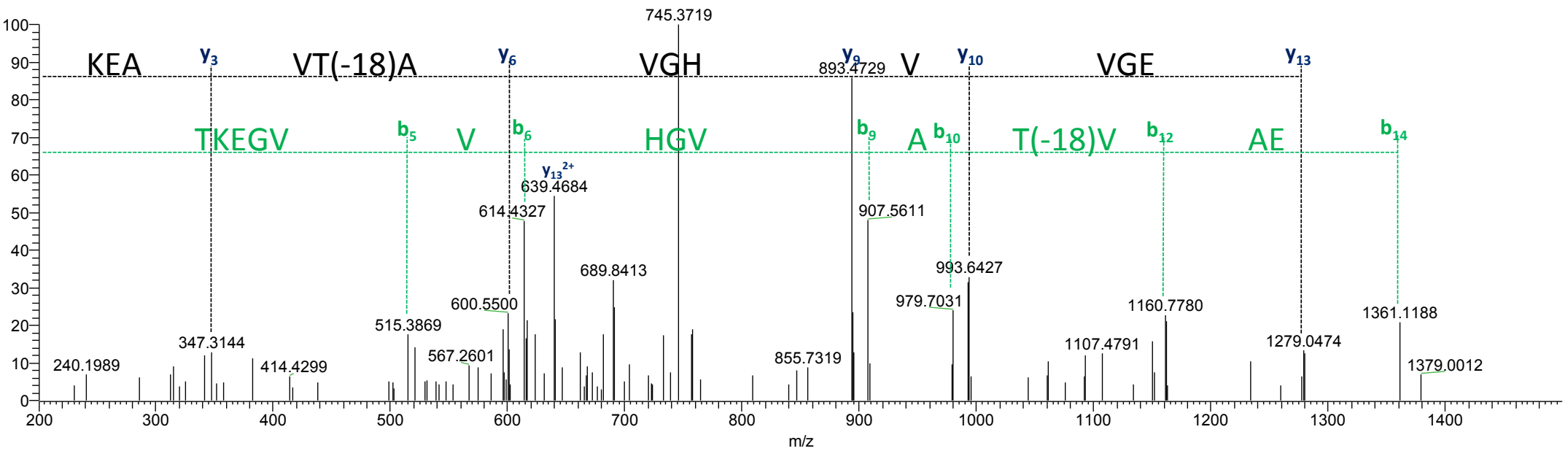




\section{(K)TKEQVTNVGGAVVTGV $T_{75}{ }^{\text {AMPP }}$ AVAQK(T)}
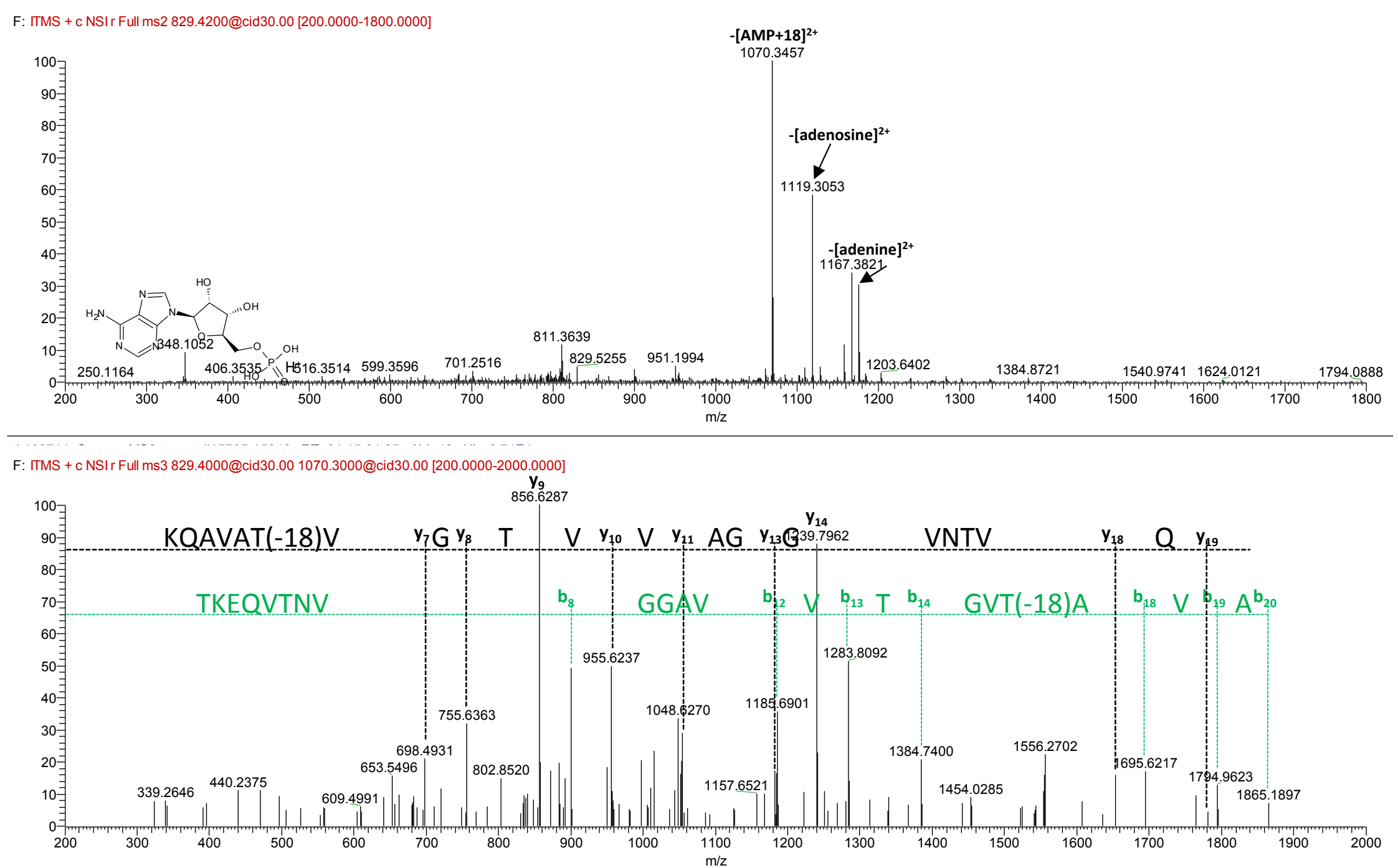
A:

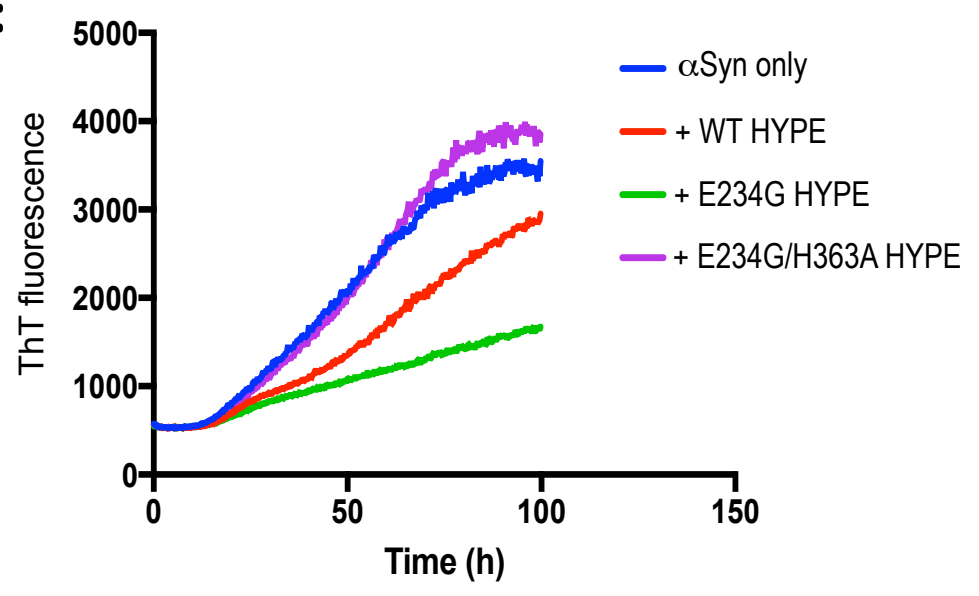

C:

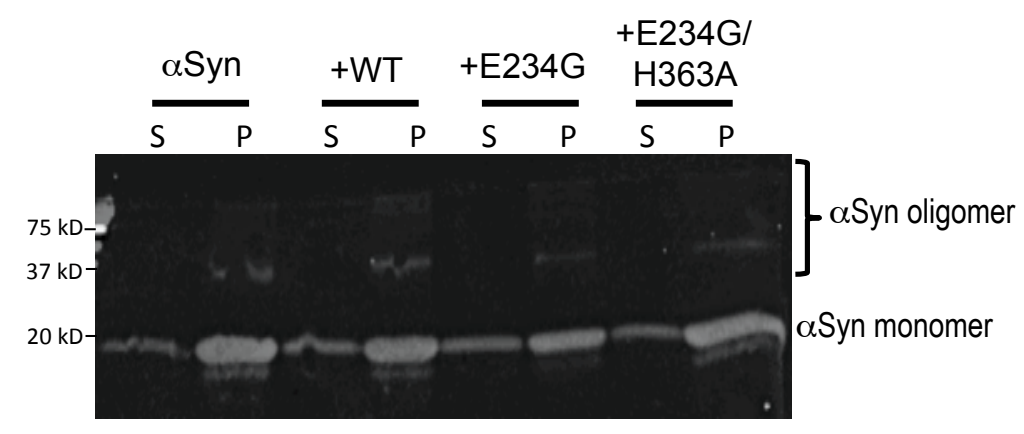

D:

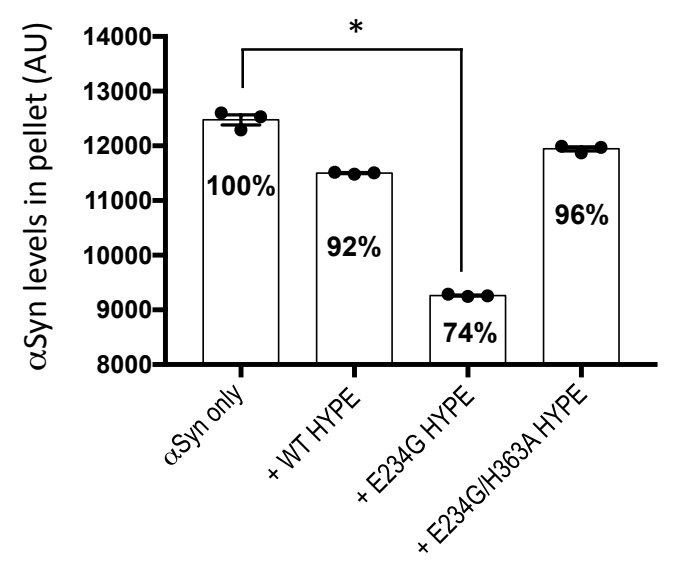

B:

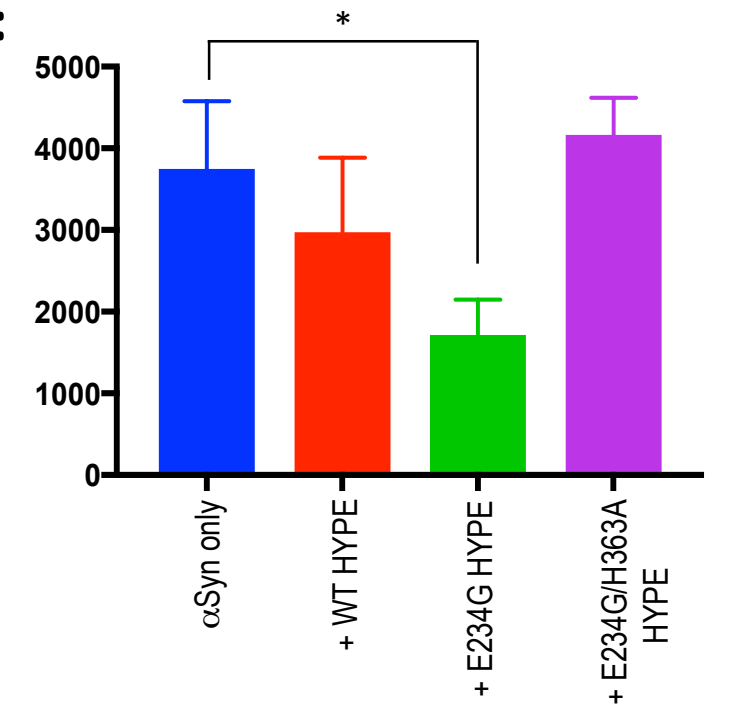

E:

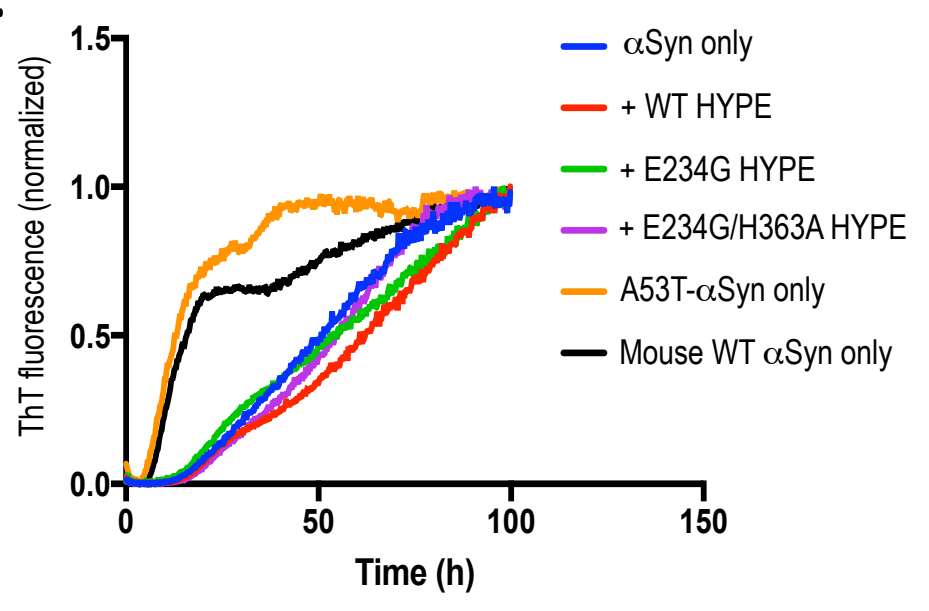


A

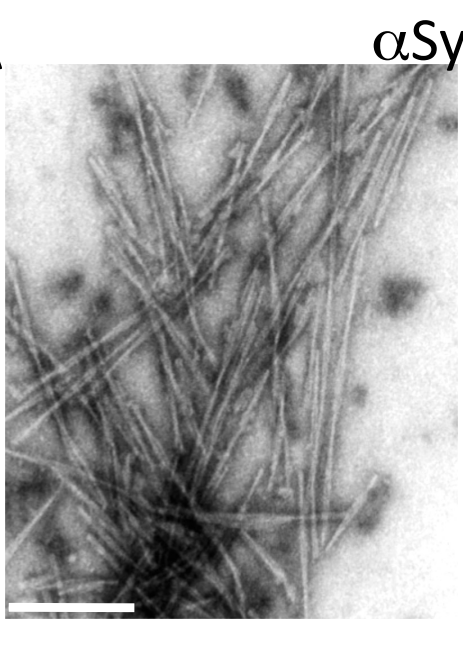

aSyn only

C
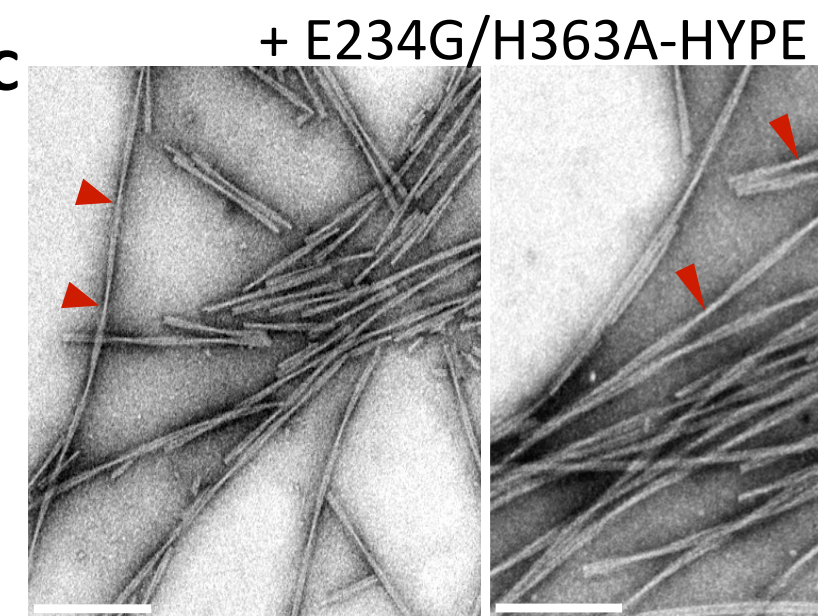

$-200 \mathrm{~nm}$

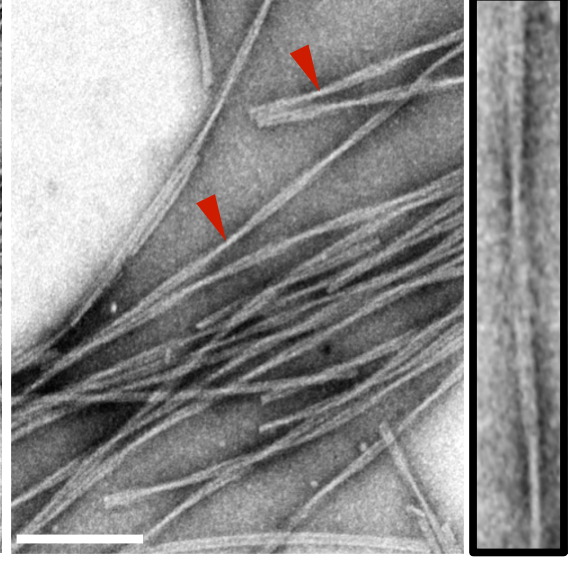

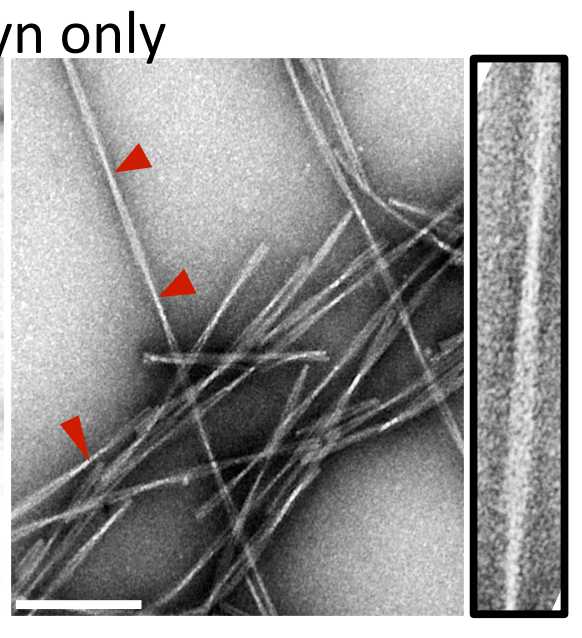

+ WT-HYPE

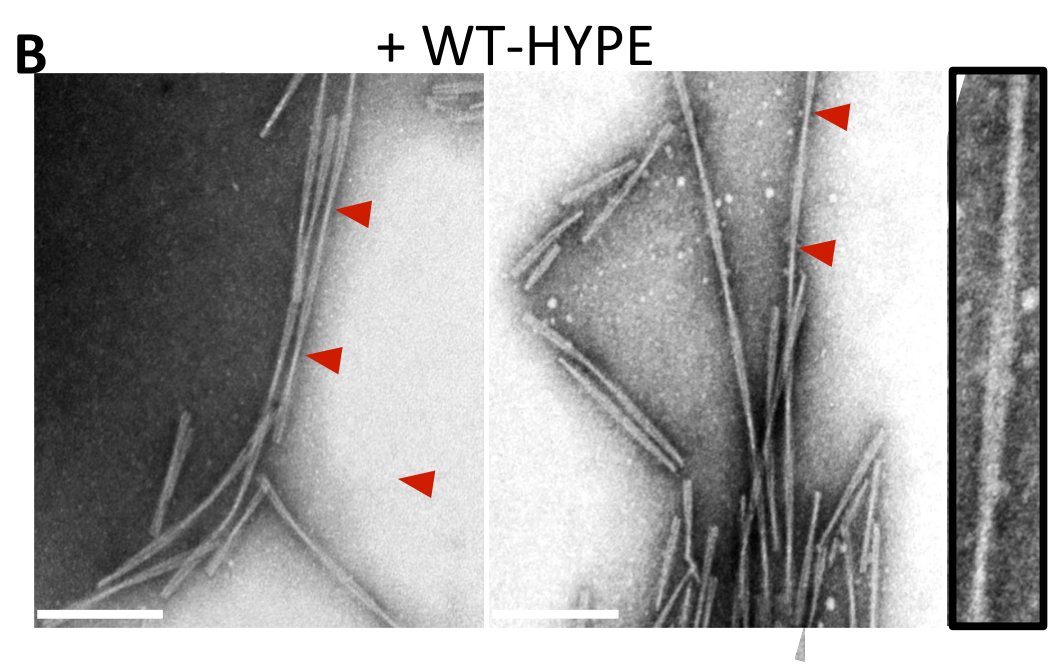

D

+ E234G-HYPE
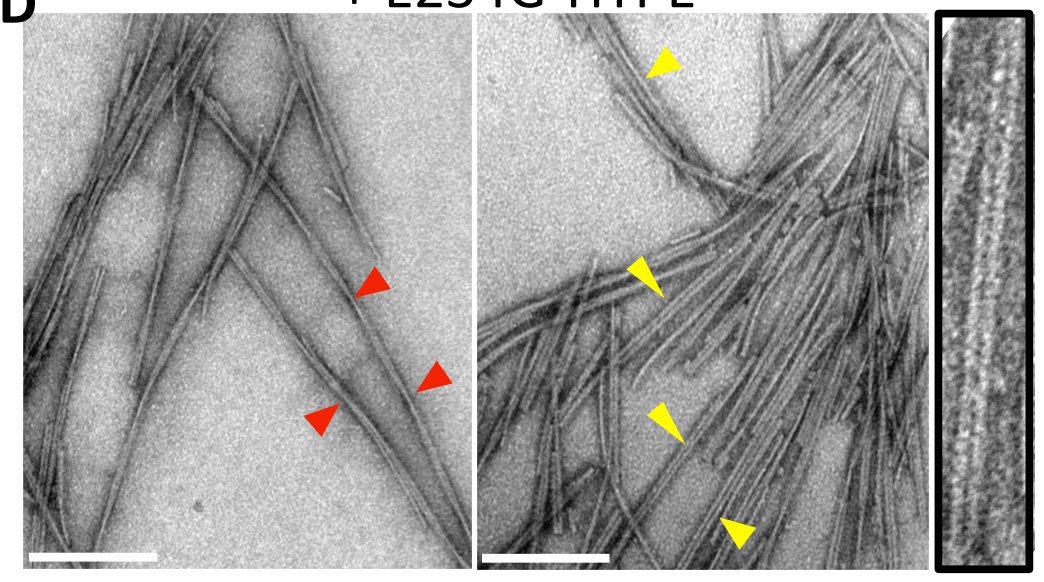


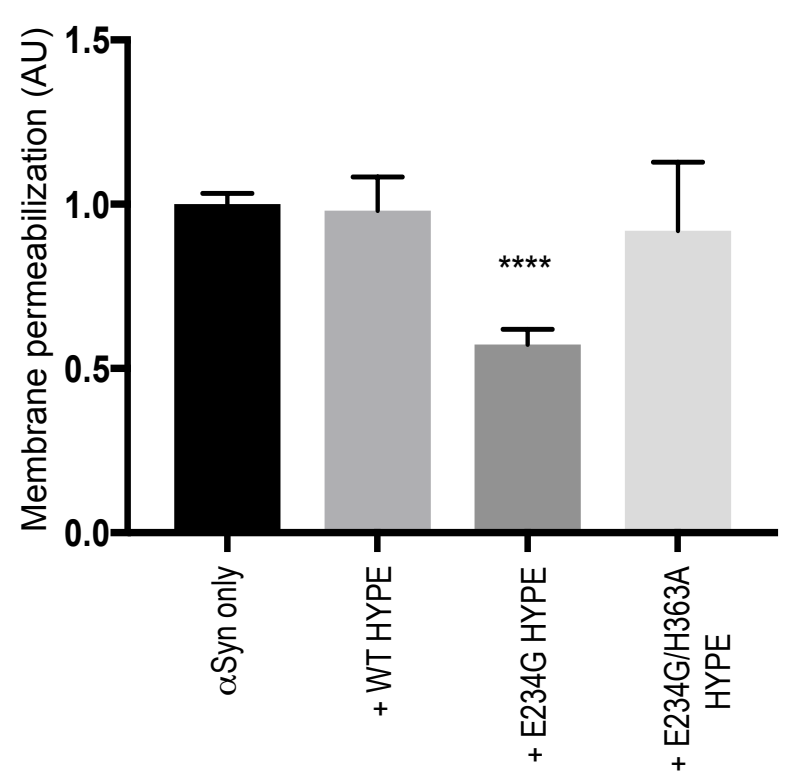


Sanyal, Dutta et al., 2019-Supplementary Figure 1

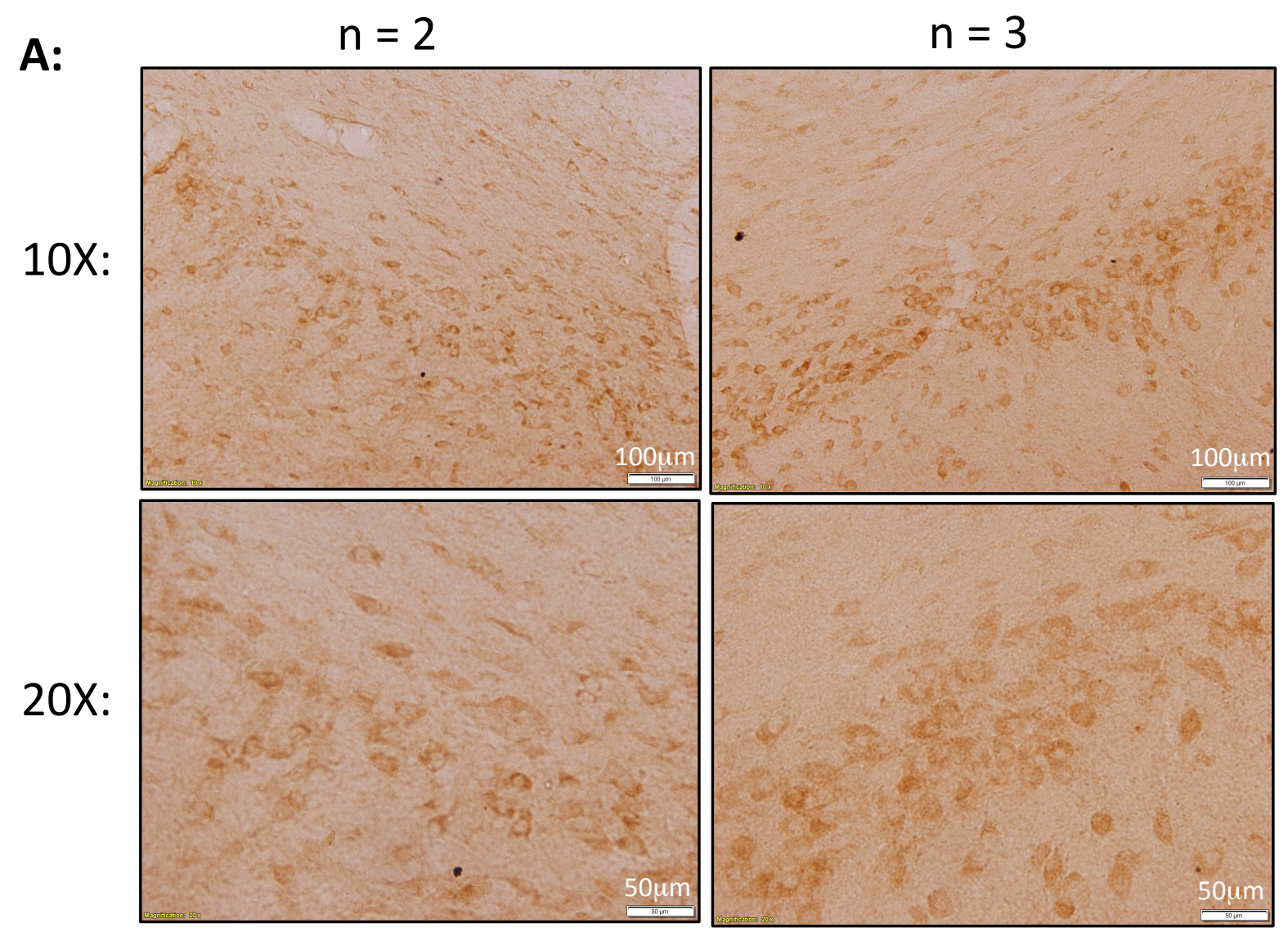


Sanyal, Dutta et al., 2019-Supplementary Figure 2
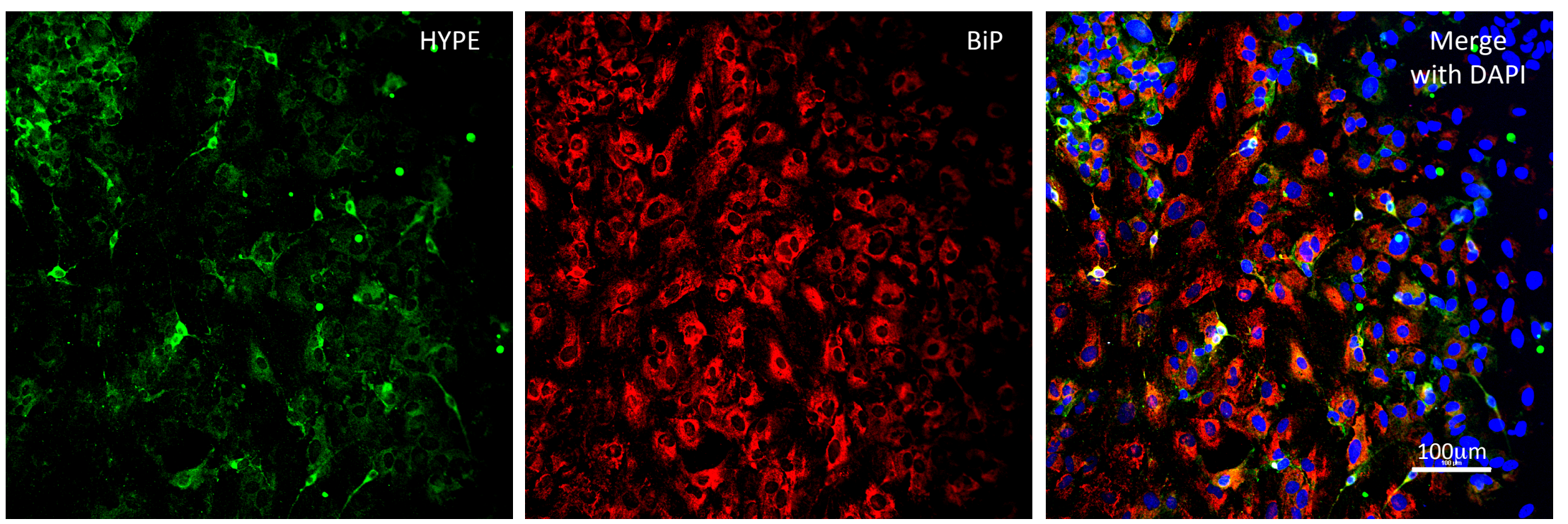
Sanyal, Dutta et al., 2019 - Supplementary Figure 3

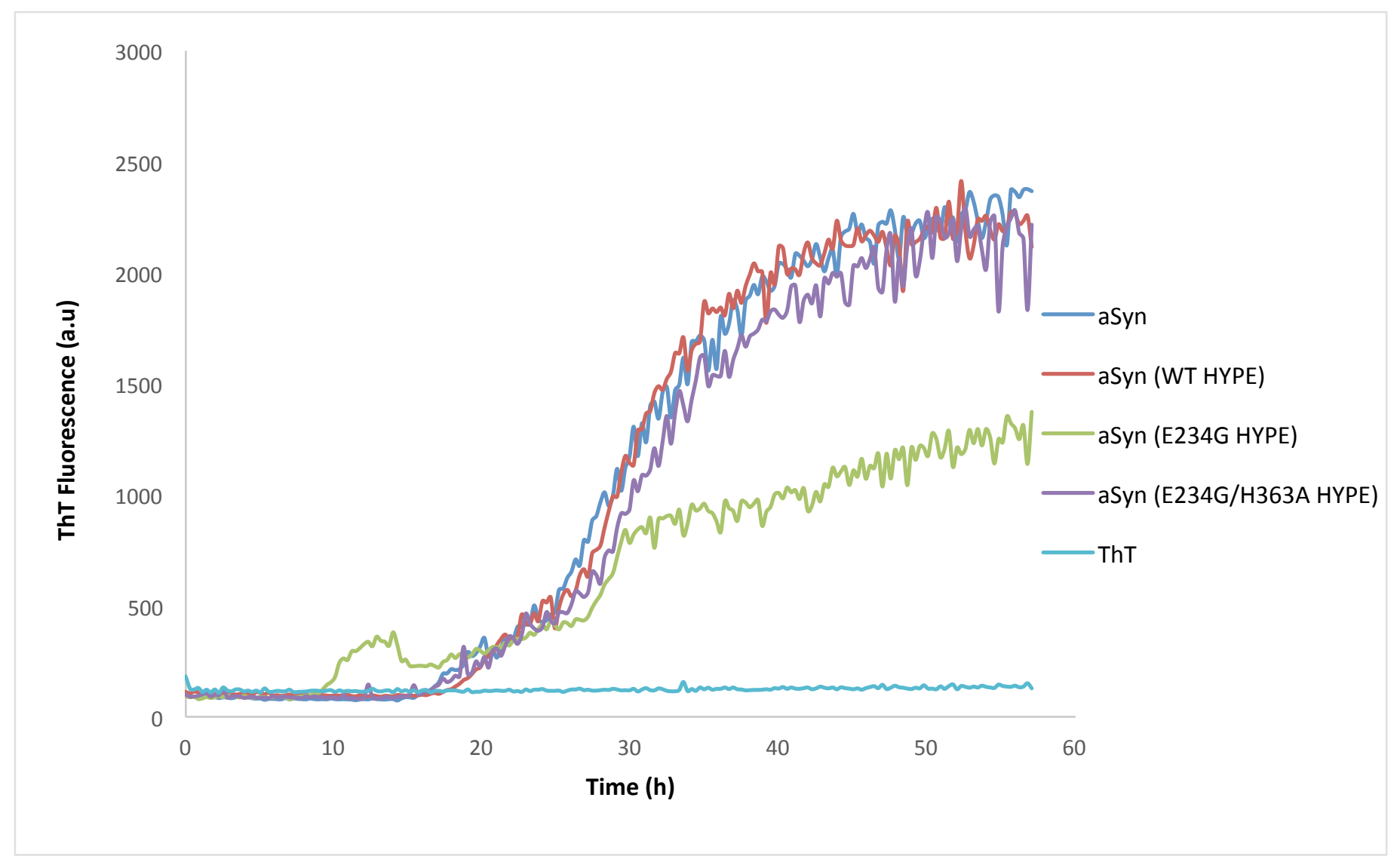

